\title{
LotuS2: An ultrafast and highly accurate tool for amplicon sequencing analysis
}

Ezgi Özkurt ${ }^{1,2}$, Joachim Fritscher ${ }^{1,2}$, Nicola Soranzo², Duncan Y. K. Ng${ }^{1}$, Robert P. Davey² Mohammad Bahram ${ }^{3,4}$, Falk Hildebrand ${ }^{1,2, \#}$

${ }^{1}$ Gut Microbes \& Health, Quadram Institute Bioscience, Norwich Research Park, Norwich, Norfolk, NR4 7UQ, UK

${ }^{2}$ Earlham Institute, Norwich Research Park, Norwich, Norfolk, NR4 7UZ, UK

${ }^{3}$ Department of Ecology, Swedish University of Agricultural Sciences, Uppsala, Ulls väg 16, 756 51 Sweden

${ }^{4}$ Institute of Ecology and Earth Sciences, University of Tartu, Tartu, 40 Lai St. Estonia

\# Corresponding author: falk.hildebrand@quadram.ac.uk

\section{Abstract}

Background: Amplicon sequencing is an established and cost-efficient method for profiling microbiomes. However, many available tools to process this data require both bioinformatics skills and high computational power to process big datasets. Furthermore, there are only few tools that allow for long read amplicon data analysis. To bridge this gap, we developed the LotuS2 (Less OTU Scripts 2) pipeline, enabling user-friendly, resource friendly, and versatile analysis of raw amplicon sequences.

Results: In LotuS2, six different sequence clustering algorithms as well as extensive pre- and post-processing options allow for flexible data analysis by both experts, where parameters can be fully adjusted, and novices, where defaults are provided for different scenarios.

We benchmarked three independent gut and soil datasets, where LotuS2 was on average 29 times faster compared to other pipelines - yet could better reproduce the alpha- and betadiversity of technical replicate samples. Further benchmarking a mock community with known taxa composition showed that, compared to the other pipelines, LotuS2 recovered a higher fraction of correctly identified genera and species ( $98 \%$ and $57 \%$, respectively). At ASV/OTU level, precision and F-score were highest for LotuS2, as was the fraction of correctly reconstructed $16 \mathrm{~S}$ sequences.

Conclusion: LotuS2 is a lightweight and user-friendly pipeline that is fast, precise and streamlined. High data usage rates and reliability enable high-throughput microbiome analysis in minutes.

Availability: LotuS2 is available from GitHub, conda or via a Galaxy web interface, documented at http://lotus2.earlham.ac.uk/.

36

Keywords: microbiome, short read sequencing, amplicon data analysis, 16S rRNA, ITS

\section{BACKGROUND:}

40 The field of microbiome research has been revolutionized in the last decade, owing to

41 methodological advances in DNA-based microbial identification. Amplicon sequencing (also

42 known as metabarcoding) is one of the most commonly used techniques to profile microbial 
43 communities based on targeting and amplifying phylogenetically conserved genomic regions

44 such as the 16S/18S ribosomal RNA (rRNA) or internal transcribed spacers (ITS) for

45 identification of bacteria and eukaryotes (esp. Fungi), respectively [1,2]. The popularity of

46 amplicon sequencing has been growing due to its broad applicability, ease-of-use, cost-

47 efficiency, streamlined analysis workflows as well as specialist applications such as low

48 biomass sampling [3].

50 Alas, amplicon sequencing comes with several technical challenges. These include primer

51 biases [4], chimeras occurring in PCR amplifications [5], rDNA copy number variations [6] and

52 sequencing errors that frequently inflate observed diversity [7]. Although modern read error

53 corrections can significantly decrease artifacts of sequencing errors [8], the taxonomic

54 resolution is limited to the genus or at best to species level $[9,10]$. To process amplicon

55 sequencing data from raw reads to taxa abundance tables, several pipelines have been

56 developed, such as mothur [11], QIIME 2 [12], DADA2 [8] or LotuS [13]. These pipelines differ in

57 their data processing and sequence clustering strategies, reflected in differing execution speed

58 and resulting amplicon interpretations [13,14].

60 Here we introduce Lotus2, designed to improve reproducibility, accuracy and ease of amplicon

61 sequencing analysis. LotuS2 offers a completely refactored installation, including a web

62 interface that is freely deployable on Galaxy clusters. During development, we focused on all

63 steps of amplicon data analysis, including processing raw reads to abundance tables as well as

64 improving taxonomic assignments and phylogenies of Operational Taxonomic Units (OTUs) or

65 Amplicon Sequencing Variants (ASVs) at the highest quality with the latest strategies available.

66 Pre- and post-processing steps were further improved compared to the predecessor "LotuS1":

67 the read filtering program sdm (simple demultiplexer) and taxonomy calculation program LCA

68 (least common ancestor) were refactored and parallelized in $\mathrm{C}_{++}$. LotuS2 uses a 'seed 
extension' algorithm that improves the quality and length of OTU/ASV representative DNA sequences. We integrated numerous features such as additional sequence clustering options (DADA2, UNOISE3, VSEARCH and CD-HIT), advanced read quality filters based on probabilistic and Poison binomial filtering and curated ASVs/OTUs diversity and abundances (LULU, UNCROSS2, ITSx, host DNA filters). LotuS2 can also be integrated in complete workflows, e.g. the microbiome visualization-centric pipeline CoMA [15] uses LotuS1/2 at its core to estimate taxa abundances. Here, we evaluated LotuS2 in reproducing microbiota profiles in comparison to contemporary amplicon sequencing pipelines. We found that LotuS2 consistently reproduces microbiota profiles more accurately, using three independent datasets, and reconstructs a mock community with the highest overall precision.

\section{MATERIALS AND METHODS:}

82 Design Philosophy of LotuS2

83 Overestimating observed diversity is one of the central problems in amplicon sequencing,

84 mainly due to sequencing errors $[7,16]$. The second read pair from Illumina paired-end

85 sequencing is generally lower in quality [17] and can contain more errors than predicted from

86 Phred quality scores alone [18,19]. Additionally, merging reads can introduce chimeras due to

87 read pair mismatches [20]. The accumulation of errors over millions of read pairs can impact

88 observed biodiversity, so essentially is a multiple testing problem. To avoid overestimating

89 biodiversity, LotuS2 uses a relatively strict read filtering during the error-sensitive sequence

90 clustering step. This is based on i) 21 quality filtering metrics (average quality, homonucleotide

91 repeats, removal of reads without amplicon primers, etc), ii) probabilistic and Poisson binomial

92 read filtering $[17,21]$, iii) filtering reads that cannot be dereplicated (clustered at $100 \%$ nucleotide

93 identity) either within or between samples and iv) using only the first read pair from paired-end

94 Illumina sequencing platforms. These reads are termed "high-quality" reads in the pipeline 
description and are clustered into OTUs/ASVs, using one of the sequence clustering programs

96 (Figure 1B).

97 However, filtered out "mid-quality" sequences are partly recovered later in the pipeline, during

98 the seed extension step. LotuS2 will reintroduce reads failing dereplication thresholds or being

99 of "mid-quality" by mapping these reads back onto high-quality OTUs/ASVs if matching at $\geq$

$10097 \%$ sequence identity. In the "seed extension" step, the optimal sequence representing each

101 OTU/ASV is determined by comparing all (raw) reads clustered into each OTU/ASV. The best

102 read (pair) is then selected based on the highest overall similarity to the consensus OTU/ASV,

103 quality and length that, in the case of paired read data, can then be merged. Thereby, the seed

104 extension step enables more reads to be included in taxa abundance estimates, as well as

105 enabling longer ASV/OTU representative sequences to be used during taxonomic classifications

106 and the reconstruction of a phylogenetic tree.

Implementation of LotuS2

Installation - LotuS2 can be accessed either through major software repositories such as i)

111 Bioconda, ii) as a Docker image or iii) GitHub (accessible through http://lotus2.earlham.ac.uk/)

112 (Figure 1A). The GitHub version comes with an installer script that downloads the required

113 databases and installs and configures LotuS2 with its dependencies. Alternatively, we provide

114 iv) a wrapper for Galaxy [22] allowing installation of LotuS2 on any Galaxy server from the

115 Galaxy ToolShed. LotuS2 is already available to use for free on the UseGalaxy.eu server

116 (https://usegalaxy.eu/), where raw reads can be uploaded and analysed (Supp. Figure 1).

117 While LotuS2 is natively programmed for Unix (Linux, macOS) systems, other operating

118 systems are supported through the Docker image or the Galaxy web interface.

119 Input - LotuS2 is designed to run with a single command, where the only essential flags are the

120 path to input files (fastq(.gz), fna(.gz) format), output directory and mapping file. The mapping 
121 file contains information on sample identifiers, demultiplexing barcodes or file paths to already

122 demultiplexed files and can be either automatically generated or provided by the user. The

123 sequence input is flexible, allowing simultaneous demultiplexing of read files and/or integration

124 of already demultiplexed reads.

125 LotuS2 is highly configurable, enabling user-specific needs beyond the well-defined defaults.

126 There are 63 flags that can be user-modified, including dereplication filtering thresholds (-

127 derepMin), sequencing platform (-p), amplicon region (-amplicon_type), or OTU/ASV

128 postprocessing (e.g. -LULU option to remove erroneous OTUs/ASVs [23]). In addition, read

129 filtering criteria can be controlled in 32 detailed options via custom config files (defaults are

130 provided for Illumina MiSeq, hiSeq, novaSeq, Roche 454, PacBio HiFi).

131

132 Output - The primary output is a set of tab-delimited OTU/ASV count tables, the phylogeny of

133 OTUs/ASVs, their taxonomic assignments and corresponding abundance tables at different

134 taxonomic levels. These are summarized in .biom [24] and phyloseq objects [25], that can be

135 loaded directly by other software for downstream analysis.

136 Furthermore, a detailed report of each processing step can be found in the log files which

137 contain commands of all used programs (including citations and versions) with relevant

138 statistics. We support and encourage users to conduct further analysis in statistical

139 programming languages such as R, Python or Matlab and using analysis packages such as

140 phyloseq [25], documented in tutorials at http://lotus2.earlham.ac.uk/. .

142 Pipeline workflow - Most of LotuS2 is implemented in PERL 5.1; computational or memory

143 intensive components like simple demultiplexer (sdm) and LCA (least common ancestor) are

144 implemented in $\mathrm{C}++$ (see Figure 1B for pipeline workflow). Demultiplexing, quality filtering and 
145 dereplication of reads is implemented in sdm. Taxonomic postprocessing is implemented in

146 LCA. Six sequence clustering methods are available: UPARSE [17], UNOISE3 [26], CD-HIT

147 [27], SWARM [28], DADA2 [8] or VSEARCH [29].

148 In the "seed extension" step, a unique representative read of a sequence cluster is chosen,

149 based on quality and merging statistics. Each sequence cluster, termed ASVs in the case of

150 DADA2, OTUs otherwise ${ }^{1}$, is represented by a high confidence DNA sequence (see Design

151 Philosophy of LotuS2 for more information).

152 OTUs/ASVs are further postprocessed to remove chimeras, either de novo and/or reference

153 based using the program UCHIME3 [30] or VSEARCH-UCHIME [29]. By default, ITS sequences

154 are extracted using ITSx [31]. Highly resolved OTUs/ASVs are then curated based on sequence

155 similarity and co-occurrence patterns, using LULU [23]. False-positive OTU/ASV counts can be

156 filtered using the UNCROSS2 algorithm [32]. OTUs/ASVs are by default aligned against the

157 phiX genome, a synthetic genome often included in Illumina sequencing runs, using Minimap2

158 [33]; these OTUs/ASVs are subsequently removed. Additionally, the user can filter for host

159 contamination by providing custom genomes (e.g., human reference), as host genome reads

160 are often misclassified as bacterial 16S by existing pipelines [3].

161 Each OTU/ASV is taxonomically classified, using either RDP classifier [34], SINTAX [35] or by

162 alignments to reference database(s), using the custom "LCA" (least common ancestor) $\mathrm{C}++$

163 program. Alignments of OTUs/ASVs with either Lambda [36], BLAST [37], VSEARCH [29], or

164 USEARCH [38] are compared against a user-defined range of reference databases. These

165 databases cover the 16S, 18S, 23S, 28S rRNA gene and ITS region, by default a Lambda

166 alignment against the SILVA database is used [39]. Other databases bundled with LotuS2

167 include Greengenes [40], HITdb [41], PR2 [42], beetax (bee gut-specific taxonomic annotation)

168 [43], UNITE (fungal ITS database) [44], or users can provide reference databases (a fasta file ${ }^{1}$ Note that UNOISE3 uses the term zero-range OTUs (zOTUs); for brevity, this is omitted throughout the
text. 
and a tab-delimited taxonomy file). These databases can be used by themselves, or in conjunction. From mappings against one or several reference databases, the least common

171 ancestor for each OTU/ASV is calculated using LCA. Priority is given to deeply resolved

172 taxonomies, sorted by the earlier listed reference databases. For reconstructing phylogenetic

173 trees, multiple sequence alignments for all OTUs/ASVs are calculated with either MAFFT [45] or

174 Clustal $\Omega$ [46]; from these a maximum likelihood phylogeny is constructed using either fasttree2

175 [47] or IQ-TREE 2 [48].

176

177

178

179

180

181

182

183

184

185

Our benchmarking scripts are available at https://github.com/ozkurt/lotus2 benchmarking (see

187 Supp. Text). Several sequence cluster algorithms were benchmarked, for LotuS2: DADA2 [8], UPARSE [17], UNOISE3 [26], CD-HIT [27] and VSEARCH [29]; for QIIME 2: DADA2 and Deblur [49]; DADA2 supporting natively only DADA2 clustering; for mothur: OptiClust; and for LotuS1: UPARSE. For taxonomic classification, SILVA138.1 [39], was used in all pipelines.

\section{Benchmarking amplicon sequencing pipelines}

To benchmark the computational performance and reproducibility, we compared LotuS2's performance to commonly used amplicon sequencing pipelines including mothur [11], DADA2 [8], and QIIME 2 [12]. We relied, where possible, on default options or standard operating procedure (SOPs) provided by the respective developers (mothur:

https://mothur.org/wiki/miseq sop/; QIIME 2: https://docs.qiime2.org/2021.11/tutorials/movingpictures/ and DADA2: https://benijneb.github.io/dada2/tutorial.html). DADA2 cannot demultiplex raw reads and in these cases, LotuS2 demultiplexed raw reads were used as DADA2 input.

ITS amplicons are clustered with CD-HIT, UPARSE and VSEARCH and filtered by default using ITSx [31] in LotuS2. ITSx identifies likely ITS1, 5.8S and ITS2 and full-length ITS sequences, 
and sequences not within the confidence interval are discarded in LotuS2. In analogy, QIIME 2-

194 DADA2 uses q2-ITSxpress [50] that also removes unlikely ITS sequences.

196 Error profiles during ASV clustering were inferred separately for the samples sequenced in 197 different MiSeq runs during DADA2 and Deblur clustering in all pipelines. We truncated the 198 reads into the same length (200 bases, default by LotuS2) in all pipelines while analysing the 199 datasets. Primers were removed from the reads, where supported by a pipeline.

\section{Measuring computational performance of amplicon sequencing pipelines}

202 When benchmarking pipelines, processing steps were separated into 5 categories in each

203 tested pipeline: a) Pre-processing (demultiplexing if required, read filtering, primer removal and

204 read merging for QIIME 2-Deblur), b) sequence clustering (clustering + refining of the clusters

205 and denoising for QIIME 2-DADA2, c) OTU/ASV taxonomic assignment, d) construction of a

206 phylogenetic tree (the option is available only in mothur, QIIME 2 and LotuS2) and e) removal of

207 host genome (the option is available only in QIIME 2 and LotuS2). In mothur, sequence

208 clustering and taxonomic assignment times were added since these pipeline commands are

209 entangled (https://mothur.org/wiki/miseq sop/).

\section{Data used in benchmarking pipeline performance}

212 Four datasets with different sample characteristics (e.g., compositional complexity, target gene 213 and region, amplicon length) were analysed: i) Gut-16S dataset [13]: 16S rRNA gene amplicon

214 sequencing of 40 human faecal samples in technical replicates that were sequenced in separate

215 MiSeq runs, totalling 35,412,313 paired-end reads. Technical replicates were created by

216 extracting DNA twice from each faecal sample. Since the Illumina runs were not demultiplexed,

217 pipelines had to demultiplex these sequences, if available. ii) Soil-16S dataset: 16S rRNA gene

218 amplicon sequencing of two technical replicates (single DNA extraction per sample) from 50 soil 
219 samples, that were sequenced in separate MiSeq runs, totalling 11,820,327 paired-end reads.

220 PCR reactions were conducted using the $16 \mathrm{~S}$ rRNA region primers 515F

221 (GTGYCAGCMGCCGCGGTAA) and 926R (GGCCGYCAATTYMTTTRAGTTT). The soil-16S

222 dataset was already demultiplexed, requiring pipelines to work with paired FASTQ files per

223 sample. iii) Soil-ITS dataset: ITS amplicon sequencing of 50 technical replicates of soil samples

224 (single DNA extraction per sample), sequenced in two independent Illumina MiSeq runs,

225 totalling 6,006,089 paired-end reads. ITS region primers gITS7ngs_201

226 (GGGTGARTCATCRARTYTTTG) and ITS4ngsUni_201 (CCTSCSCTTANTDATATGC) [51]

227 were used to amplify DNA extracted from soil samples. The soil-ITS dataset was already

228 demultiplexed.

229 iv) Mock dataset [52]: A microbial mock community with known species composition, mock-16

230 [52]. The mock dataset comprised a total of 59 strains of Bacteria and Archaea, representing 35

231 bacterial and 8 archaeal genera. The mock community was sequenced on an Illumina MiSeq

232 (paired-end) by targeting the V4 region of the 16S rRNA gene using the primers 515F

233 (GTGCCAGCMGCCGCGGTAA) and 806R (GGACTACHVGGGTWTCTAAT) [52]. This dataset

234 was demultiplexed and contained 593,868 paired reads.

Benchmarking the computational performance of amplicon sequencing pipelines

236 To evaluate the computational performance of LotuS2 in comparison to QIIME 2 [12], DADA2

237 [8], and the last released version of LotuS [13] (v1.62 from Jan 2020; called LotuS1 here), all

238 pipelines were run with 12 threads on a single computer free of other workloads (CPU: Intel(R)

239 Xeon(R) Gold $6130 \mathrm{CPU} @ 2.10 \mathrm{GHz}, 32$ cores, 375 GB RAM). To reduce the influence of

240 network latencies on pipeline execution, all temporary, input, and output data were stored on a

241 local SSD. Each pipeline was run three times consecutively to account for pre-cached data and

242 to obtain average execution time and maximum memory usage. To calculate the fold

243 differences in execution speed between pipelines, the average time of all LotuS2 runs was 
244 divided by average QIIME 2, mothur and DADA2, where used in each of the three non-mock

245 datasets. The average of these numbers was used to estimate the average speed advantage of

246 LotuS2.

248 Benchmarking reproducibility of amplicon sequencing pipelines

249 Technical replicates of the soil and gut samples were used to estimate the reproducibility of the 250 microbial community composition between replicates. This was measured by calculating beta 251 and alpha diversity differences between technical replicate samples. To calculate beta diversity, 252 either Jaccard (measuring presence/absence of OTUs/ASVs) or Bray-Curtis dissimilarity 253 (measuring both presence/absence and abundances of OTUs/ASVs) were computed between 254 technical replicate samples. Before computing Bray-Curtis distances, abundance matrices were 255 normalized. Jaccard distances between samples were calculated by first rarefying abundance 256 matrices to an equal number of reads (to the size of the first sample having $>1000$ read counts)

257 per sample using RTK [53]. Significance of pairwise comparisons of the pipelines in beta

258 diversity differences was calculated using the ANOVA test where Tukey's HSD (honest

259 significant differences) test was used as a post hoc test in R.

260 To calculate alpha diversity, abundance data were first rarefied to an equal number of reads per 261 sample. Significance of each pairwise comparison in alpha diversity was calculated based on a 262 paired Wilcoxon test, pairing technical replicates.

\section{Analysis of the mock community}

265 We used an already sequenced mock community [52] of known relative composition and with

266 sequenced reference genomes available. Firstly, taxonomic abundance tables (taxonomic

267 assignments based on SILVA 138.1 [39] in all pipelines) were compared to the expected

268 taxonomic composition of the sequenced mock community. Precision was calculated as

$269(\mathrm{TP} /(\mathrm{TP}+\mathrm{FP}))$, recall as $(\mathrm{TP} /(\mathrm{TP}+\mathrm{FN}))$ and F-score as $\left(2^{*}\right.$ precision*recall/(precision+recall)), TP 
270 (true positive) being taxa present in the mock and correctly identified as present, FN (false

271 negative) being taxa present in the mock but not identified as present and FP (false positive)

272 being taxa absent in the mock but identified as present. The fraction of read counts assigned to

273 true positive taxa was calculated based on the sum of the relative abundance of all true positive

274 taxa. These scores were calculated at different taxonomic levels.

275 Secondly, we investigated the precision of reconstructed 16S rRNA nucleotide sequences,

276 representing each OTU or ASV, by calculating the nucleotide similarity between ASVs/OTUs

277 and the known reference 16 S rRNA sequences. To obtain the nucleotide similarity, we aligned

278 ASV/OTU DNA sequences from tested pipelines via BLAST to a custom reference database

279 that contained the 16S rRNA gene sequences from the mock community

280 (https://github.com/caporaso-lab/mockrobiota/blob/master/data/mock-16/source/expected-

281 sequences.fasta), using the -taxOnly option from LotuS2. The BLAST \% nucleotide identity was

282 subsequently used to calculate the best matching $16 \mathrm{~S}$ rRNA sequence per ASV/OTU.

RESULTS

286 We analysed four datasets to benchmark the computational performance and reliability of the

287 pipelines. The datasets consisted either of technical replicates (gut-16S, soil-16S, soil-ITS) or a

288 mock community. Technical replicates were used to evaluate the reproducibility of community

289 structures and were chosen to represent different biomes (gut, soil), using different 16S rRNA

290 amplicon primers (gut-16S, soil-16S) or ITS sequences (soil-ITS) as well as a synthetic mock

291 community of known composition.

292

\section{Computational performance and data usage}

294 The complete analysis of the gut-16S dataset was fastest in LotuS2 (on average 35, 12, 9 and 2953.8 times faster than mothur, QIIME 2-DADA2, QIIME 2-DEBLUR and native DADA2, 
respectively, Figure $\mathbf{2 A}$ ). Note that DADA2 could not demultiplex the dataset, the average of

297 LotuS2 and QIIME2 demultiplexing times were used instead. LotuS2 was also faster in the

298 analysis of the soil-16S dataset compared to the other tested pipelines $(5.7,3.5,3.5$ times faster

299 than DADA2, QIIME 2-DADA2 and QIIME 2-DEBLUR, respectively, Figure 2B). The difference

300 in speed between LotuS2 and QIIME 2 was more pronounced in the analysis of the soil-ITS

301 dataset, where LotuS2 was on average 69 times faster than QIIME 2 and DADA2 (Figure 2C).

302 LotuS2 also outperformed other pipelines in the case of the gut-16S dataset (on average

303 LotuS2 was 15 times faster) compared to the soil dataset (average 4.2). This difference stems

304 mainly from the demultiplexing step, where LotuS2 is significantly faster. The sequence

305 clustering step was fastest using the UPARSE algorithm, i.e. an average 60-fold faster than

306 sequence clustering in other pipelines. Averaged over these three datasets, LotuS2 was 29

307 times faster than other pipelines.

308 Taxonomic classification of OTUs/ASVs was also faster in LotuS2 ( 5 times faster for gut-16S,

3092 times for soil-16S). However, this strongly depends on the total number of OTUs/ASVs for all

310 pipelines. For example, the default naïve-Bayes classifier [54] in QIIME 2 is faster relative to the

311 number of OTUs/ASVs, compared to LotuS2 taxonomic assignments in this benchmark.

312 Nevertheless, the LotuS2 default taxonomic classification is via RDP classifier [34], and

313 alternatively, the SINTAX [35] classifier could be used, both of which are significantly faster than

314 the here presented Lambda LCA against the Silva reference database.

315 Compared to LotuS1, LotuS2 was on average 3.2 times faster, likely related to refactored $\mathrm{C}++$

316 programs that can take advantage of multiple CPU threads (Figure 2A-B).

317 In its fastest configuration (using "UPARSE" option in clustering, "RDP" to assign taxonomy), the

318 gut and soil 16S rRNA datasets can be processed with LotuS2 in under 20 mins and 12 mins,

319 using $<10 \mathrm{~GB}$ of memory and 4 CPU cores.

320 Despite using similar clustering algorithms (e.g. DADA2 is used in DADA2, QIIME 2 and

321 LotuS2), the tested pipelines apply different pre- and post-processing algorithms to raw 
sequence reads and clustered ASVs and OTUs, leading to differing ASV/OTU numbers and retrieved reads (the total read count in the ASV/OTU abundance matrix) (Supp. Table 1 and

324 Figure 2D-F). DADA2 typically estimated the highest number of ASVs, but the number of 325 retrieved reads varied strongly between datasets. QIIME 2-DADA2 estimated fewer ASVs than

326 DADA2, but more ASVs than LotuS2-DADA2, although mapping fewer reads than LotuS2.

327 Although retrieving a smaller number of reads, QIIME 2-Deblur reported comparable numbers of 328 ASVs to LotuS2, despite the differences in clustering algorithms. mothur performed differently in 329 the gut-16S and soil-16S datasets, where it estimated either the highest number of OTUs or 330 could not complete the analysis since all the reads being filtered out, respectively. Overall,

331 LotuS2 often reported the fewest ASVs/OTUs, while including more sequence reads in

332 abundance tables. This indicates that LotuS2 has a more efficient usage of input data while covering a larger sequence space per ASV/OTU.

\section{Benchmarking the reproducibility of community compositions}

336 Next, we assessed the reproducibility of community compositions, using gut-16S, soil-16S and 337 soil-ITS datasets comparing beta diversity between technical replicates (Bray Curtis distance, 338 BCd and Jaccard distance, Jd). We found that Jd and BCd were the lowest in LotuS2, largely 339 independent of the chosen sequence clustering algorithms and dataset. This indicates a greater 340 reproducibility of community compositions generated by LotuS2 (Figure 3A-B and Supp.

341 Figure 2). The lowest BCd and Jd were observed for UPARSE (Figure 3A-B and Supp. Figure

342 2) in both gut- and soil-16S datasets, though this was not always significant between different

343 LotuS2 runs (Supp. Table 2).

344 Even using the same clustering algorithm, LotuS2-DADA2 compositions were more

345 reproducible, compared to both QIIME 2-DADA2 and DADA2 (significant only on soil data).

346 LotuS2-DADA2 denoises by default all reads (per sequencing run) together, while in the default

347 DADA2 setup each sample is separately denoised; the latter strategy has a reduced 
computational burden but can potentially miss sequence information from rare bacteria. mothur showed poorer performance compared to other pipelines on the gut-16S dataset and did not complete on the soil data.

351 We then calculated the fraction of samples being closest in BCd distance to its technical

352 replicate for each pipeline (Figure 3D-E), simulating the process of identifying technical

353 replicates without prior knowledge. LotuS2 with UNOISE3 clustering resulted in the highest

354 fraction of samples being closest to its replicate among all samples, in both gut- and soil-16S

355 datasets while in the mothur result, technical replicates were the most unlikely to be closest to

356 their technical replicate.

357 When this comparison was made with the non-default options in LotuS2 (using different

358 dereplication parameters, deactivating LULU, using UNCROSS2 or retaining taxonomically

359 unclassified reads), BCd between the technical replicates remained largely unchanged (Supp.

360 Figure 2, Supp. Figure 3A-B and Supp. Text). However, retaining unclassified reads could

361 significantly reduce the reproducibility of LotuS2 results on the gut-16S dataset. Furthermore,

362 even starting the analysis with different read truncation lengths, LotuS2 still had the highest

363 reproducibility in both gut- and soil-16S datasets (Supp. Figure 4, Supp. Figure 5 and Supp.

364 Text).

365 Lastly, we calculated the reproducibility of reported alpha diversity between technical replicate 366 samples in both gut-16S and soil-16S datasets (Supp. Figure 6A-B). In both datasets, LotuS2

367 alpha diversity was not significantly different between technical replicates, as expected (5 of 8

368 comparisons, Wilcoxon signed-rank test), whereas, in 6 of 6 cases, QIIME 2, mothur and

369 DADA2 had significant differences in the alpha diversity between technical replicates.

370 Thus, LotuS2 showed in our benchmarks a higher data usage efficiency and higher

371 reproducibility of community compositions than QIIME 2, DADA2 and mothur. These

372 benchmarks also showed the importance of pre- and postprocessing raw reads and 
373 OTUs/ASVs, since LotuS2-DADA2 and QIIME 2-DADA2 performed better than and DADA2,

374 despite using the same clustering algorithm.

375

376 Benchmarking soil-ITS dataset

377 Unlike 16S rRNA gene amplicons, ITS amplicons typically vary greatly in length [4], requiring a

378 different sequence clustering workflow; therefore, LotuS2 uses by default CD-HIT to cluster ITS

379 sequences, and ITSx to identify plausible ITS1/2 sequences.

380 In terms of data usage, both LotuS2 and QIIME 2-DADA2 retrieved similar numbers of reads,

381 but for QIIME 2 these read counts were distributed across twice the number of ASVs (Figure

382 2F). QIIME 2-DADA2 reproduced the fungal composition significantly worse in replicate

383 samples, compared to LotuS2-UPARSE, having higher pairwise BCd (Figure $3 \mathrm{C}$ ) and Jd

384 (Supp. Figure 2H-I). However, it spanned the highest fraction of samples closest to its technical

385 replicate, although this fraction was overall very high for all the pipelines (0.978-1) (Figure 3F).

386 DADA2 performed relatively worse, yielding the highest number of ASV, lowest retrieved read

387 counts (Figure 2F), significantly the highest BCd (Figure 3C, Suppl. Table 2) between replicate

388 samples. LotuS2 had overall the lowest BCd and Jd between replicates, using both UPARSE

389 and CD-HIT clustering (Figure 3C, Supp. Figure 2H-I). Usage of CD-HIT in combination with

390 ITSx led to an increase in OTU diversity (from 947 to 1008) although read counts remained

391 mostly the same in the final output matrix and BCd was largely similar (Supp. Figure 3C). Here,

392 deactivating LULU slightly decreased reproducibility (Supp. Figure 3C).

393 Finally, we calculated the reproducibility of alpha diversity between the technical replicate

394 samples in the soil-ITS dataset (Supp. Figure 6C). All pipelines resulted in no significant

395 difference between the technical replicate samples, thus alpha diversity was highly reproducible

396 independent of the pipeline.

398 Benchmarking the dataset from the mock microbial community 
To assess how well a known community can be reconstructed in LotuS2, we used a previously sequenced 165 mock community [52] containing 43 genera and 59 microbial strains, where

401 complete reference genomes were available.

402 All pipelines performed poorly at reconstructing the community composition (Pearson R=0.43-

403 0.67, Spearman Rho=0.54-0.80, Supp. Table 3 and Supp. Figure 7), possibly related to PCR

404 biases and rRNA gene copy number variation. Therefore, we focused on the number of

405 correctly identified taxa. For this, we calculated the number of reads assigned to true taxa as

406 well as precision, recall and F-score at genus level. LotuS2-VSEARCH and LotuS2-UPARSE

407 had the highest precision, F-score and fraction of reads assigned true positive taxa, (Figure 4A

408 and Supp. Figure 8). LotuS1 had the highest recall, but low precision. When applying the same

409 tests at species level, LotuS2-DADA2 had overall the highest precision and F-score (Supp.

410 Figure 9). QIIME 2-DEBLUR had often competitive, but slightly lower, precision, recall and F-

411 scores compared to LotuS2, while mothur, QIIME 2-DADA2 and DADA2 scores were lower

412 (Figure 4A).

413 Next, we investigated which software could best reconstruct the correct OTU/ASV sequences.

414 For this, we calculated the fraction of TP OTUs/ASVs (i.e., OTUs/ASVs which are assigned to a 415 species based on the custom mock reference taxonomy) with $97 \%-100 \%$ nucleotide identity to 416 16S rRNA sequences from reference genomes in each pipeline (Figure 4B). Here, LotuS2-

417 VSEARCH and LotuS2-UPARSE reconstructed OTU sequences were most often identical to

418 the expected sequences, having $82.2 \%$ of the OTU sequences reconstructed at $100 \%$

419 nucleotide identity to reference sequences. QIIME 2-Deblur ASV sequences were of similar

420 quality, but slightly less often at 100\% nucleotide identity (78.2\%). DADA2 and QIIME 2-DADA2

421 ASV sequences were often more dissimilar to the expected reference sequences. It is

422 noteworthy that LotuS2-DADA2 did outperform these two pipelines based on the same

423 sequence clustering algorithm, likely related to the stringent read filtering and seed extension

424 step in LotuS2. 
425 The mock community consisted of 49 bacteria and 10 archaea [52], with 128 16S rRNA gene 426 copies included in their genomes. If multiple 16S copies occur within a single genome, these 427 can diverge but are mostly highly similar or even identical to each other [55]. Thus, 59 OTUs 428 would be the expected biodiversity, and $\leq 128$ ASVs. Notably, the number of mothur and QIIME 429 2-Deblur TP ASVs/OTUs exceeded this threshold ( $N=370,198$, respectively), both pipelines 430 overestimate known biodiversity. DADA2 and QIIME 2-DADA2 generated more ASVs than 431 expected per species ( $N=94,122$ respectively), but this might account for divergent within-

432 genome 16S rRNA gene copies. LotuS2 was notably at the lower end in predicted biodiversity, 433 predicting between 53-61 OTUs or ASVs in different clustering algorithms (Supp. Table 4).

434 However, these seemed to mostly represent single species, covering the present species best 435 among pipelines, as the precision at species level was highest for LotuS2 (Supp. Figure 9),

436 thus capturing species level biodiversity most accurately.

437 Based on the mock community data LotuS2 was more precise in reconstructing 16S rRNA gene 438 sequences, assigning the correct taxonomy, detecting biodiversity, and within-genome 16S 439 copies were less likely to be clustered separately using LotuS2.

\section{DISCUSSION}

442 LotuS2 offers a fast, accurate and streamlined amplicon data analysis with new features and 443 substantial improvements since LotuS1. Software and workflow optimizations make LotuS2

444 substantially faster than either QIIME 2, DADA2 and mothur. On large datasets, this advantage 445 becomes crucial for users: for example, we processed a highly diverse soil dataset consisting of $446>11$ million non-demultiplexed PacBio HiFi amplicons (26 Sequel II libraries) in 2.5 days on 16

447 CPU cores, using a single command (unpublished data). Besides being more resource and

448 user-friendly, compositional matrices from LotuS2 were more reproducible and accurate across 449 all tested datasets (gut 16 S, soil 16S, soil ITS, mock community 16 S). 
450 LotuS2 owes high reproducibility and accuracy to the efficient use of reads based on their

451 quality tiers in different steps of the pipeline. Low-quality reads introduce noise and can

452 artificially inflate observed biodiversity, i.e., the number of OTUs/ASVs [56]. Conversely, an

453 overly strict read filter will decrease sensitivity for low-abundant members of a community by

454 artificially reducing sequencing depth. To find a trade-off, LotuS2 uses only truncated, high-

455 quality reads for sequence clustering (except ITS amplicons), while the read backmapping and

456 seed extension steps restore some of the discarded sequence data.

457 Notably, OTU/ASV reconstructed with LotuS2 were the most similar (at $>99 \%$ identity) to the

458 reference, compared to other pipelines (Figure 4B). This was mostly independent of clustering

459 algorithms used, a combination of both selecting high-quality reads for sequence clustering and

460 the seed extension step, that selects a high-quality read (pair) best representing each OTU or

461 ASV. Seed extension also decouples read clustering and read merging, avoiding the use of the

462 error-prone 3' read end or second read pair during the error sensitive sequence clustering step

463 [17]. Thereby, potential length restrictions during the clustering step will not carry over to

464 computational steps benefitting from longer sequences, such as taxonomic assignments or

465 phylogeny reconstructions.

466 In conclusion, LotuS2 is a major improvement over LotuS1, representing pipeline updates that

467 accumulated over the past eight years. It offers superior computational performance, accuracy

468 and reproducibility of results, compared to the other tested pipelines. Importantly, it is

469 straightforward to install, and programmed to reduce required user time and knowledge,

470 following the idea that less is more with LotuS2.

471

472 Availability and Requirements:

473 Availability of LotuS2: Documentation, tutorials: lotus2.earlham.ac.uk, Installation via

474 bioconda: https://anaconda.org/bioconda/lotus2 
475 Galaxy wrapper (MIT licensed): https://github.com/TGAC/earlham-

476 galaxytools/tree/master/tools/lotus2 and https://toolshed.g2.bx.psu.edu/view/earlhaminst/lotus2/

477 Galaxy server: https://usegalaxy.eu/

478 Programs (GPLv3 licensed): https://github.com/hildebra/lotus2, https://github.com/hildebra/sdm,

479 https://github.com/hildebra/LCA

480 All the commands used for the benchmarking are available in

481 https://github.com/okurt/lotus2 benchmarking

482 Availability of the data:

483 Accession numbers for the datasets used for benchmarking in this study are: PRJEB49356

484 Mock-16 community is downloaded from the mockrobiota repository [52]:

485 https://s3-us-west-2.amazonaws.com/mockrobiota/latest/mock-16/mock-forward-read.fastq.gz

486 https://s3-us-west-2.amazonaws.com/mockrobiota/latest/mock-16/mock-reverse-read.fastq.gz

488 List of abbreviations:

489 OTU: Operational taxonomic unit; ASV: Amplicon sequence variant; ITS: Internal transcribed

490 spacer; TP: True positive; FN: False negative; FP: False positive; LotuS: Less OTU Scripts;

491 sdm: simple demultiplexer; LCA: least common ancestor; DADA: The Divisive Amplicon

492 Denoising Algorithm; QIIME: Quantitative Insights Into Microbial Ecology

\section{Author contributions}

496 FH programmed LotuS2, sdm and LCA with contributions from JF, EO, MB and NS. EO

497 benchmarked pipelines with help from FH and DN. Websites, Galaxy interface, conda support

498 and installation scripts for LotuS2 were implemented by FH, JF, NS and EO. EO and FH wrote 499 the manuscript with contributions from all authors. 


\section{$501 \quad$ Funding}

502 EO, FH were supported by European Research Council H2020 StG (erc-stg-948219,

503 EPYC). EO, JF, DN, FH were supported by the Biotechnology and Biological Sciences

504 Research Council (BBSRC) Institute Strategic Program Gut Microbes and Health BB/r012490/1

505 and its constituent project BBS/e/F/000Pr10355. NS and RPD are supported by the

506 Biotechnology and Biological Sciences Research Council (BBSRC), part of UK Research and

507 Innovation, Core Capability Grant BB/CCG1720/1 and the National Capability

508 BBS/E/T/000PR9814. MB was supported by the Swedish Research Councils Vetenskapsrådet

509 (grants 2017-05019 and 2021-03724) and Formas (grant 2020-00807).

\section{Acknowledgements:}

512 The authors gratefully thank numerous LotuS1 users for consistent feedback and suggestions

513 over the years, Sarah Worsley for her valuable comments on the manuscript and Stefano

514 Romano and Rebecca Ansorge for their user-comments on LotuS2. We also would like to

515 acknowledge CyVerse UK for the hosting of the LotuS2 website.

520 1. Bahram M, Hildebrand F, Forslund SK, Anderson JL, Soudzilovskaia NA, Bodegom PM, et al.

521 Structure and function of the global topsoil microbiome. Nature [Internet]. 2018;560:233-7.

522 Available from: http://www.nature.com/articles/s41586-018-0386-6

523 2. Özkurt E, Hassani MA, Sesiz U, Künzel S, Dagan T, Özkan H, et al. Seed-derived microbial 524 colonization of wild emmer and domesticated bread wheat (Triticum dicoccoides and t.

525 aestivum) seedlings shows pronounced differences in overall diversity and composition. MBio. $5262020 ; 11$. 
527 3. Bedarf JR, Beraza N, Khazneh H, Özkurt E, Baker D, Borger V, et al. Much ado about

528 nothing? Off-target amplification can lead to false-positive bacterial brain microbiome detection

529 in healthy and Parkinson's disease individuals. Microbiome [Internet]. Microbiome; 2021;9:75.

530 Available from: https://microbiomejournal.biomedcentral.com/articles/10.1186/s40168-021-

$531 \quad$ 01012-1

532 4. Tedersoo L, Anslan S, Bahram M, Põlme S, Riit T, Liiv I, et al. Shotgun metagenomes and

533 multiple primer pair-barcode combinations of amplicons reveal biases in metabarcoding

534 analyses of fungi. MycoKeys [Internet]. Pensoft Publishers; 2015 [cited 2015 May 14];10:1-43.

535 Available from: http://mycokeys.pensoft.net/articles.php?id=4852

536 5. Haas BJ, Gevers D, Earl AM, Feldgarden M, Ward D V, Giannoukos G, et al. Chimeric 16S

537 rRNA sequence formation and detection in Sanger and 454-pyrosequenced PCR amplicons.

538 Genome Res [Internet]. 2011 [cited 2014 Jul 9];21:494-504. Available from:

539 http://www.pubmedcentral.nih.gov/articlerender.fcgi?artid=3044863\&tool=pmcentrez\&rendertyp

$540 \mathrm{e}=\mathrm{abstract}$

541 6. Lee ZM-P, Bussema C, Schmidt TM. rrnDB: documenting the number of rRNA and tRNA

542 genes in bacteria and archaea. Nucleic Acids Res [Internet]. 2009 [cited 2013 Mar 6];37:D489-

543 93. Available from:

544 http://www.pubmedcentral.nih.gov/articlerender.fcgi?artid=2686494\&tool=pmcentrez\&rendertyp

$545 \mathrm{e}=$ abstract

546 7. Quince C, Lanzen A, Davenport RJ, Turnbaugh PJ. Removing noise from pyrosequenced

547 amplicons. BMC Bioinformatics [Internet]. 2011 [cited 2013 May 23];12:38. Available from:

548 http://www.pubmedcentral.nih.gov/articlerender.fcgi?artid=3045300\&tool=pmcentrez\&rendertyp

549 e=abstract

550 8. Callahan BJ, McMurdie PJ, Rosen MJ, Han AW, Johnson AJA, Holmes SP. DADA2: High-

551 resolution sample inference from Illumina amplicon data. Nat Methods [Internet]. Nature

552 Publishing Group; 2016;13:581-3. Available from: http://dx.doi.org/10.1038/nmeth.3869 
553 9. Hildebrand F. Ultra-resolution Metagenomics: When Enough Is Not Enough. mSystems

554 [Internet]. 2021;e0088121. Available from: http://www.ncbi.nlm.nih.gov/pubmed/34463579

555 10. de Oliveira Martins L, Page AJ, Mather AE, Charles IG. Taxonomic resolution of the

556 ribosomal RNA operon in bacteria: implications for its use with long-read sequencing. NAR

557 Genomics Bioinforma [Internet]. Oxford University Press; 2020;2:1-7. Available from:

558 https://academic.oup.com/nargab/article/doi/10.1093/nargab/lqz016/5625502

559 11. Schloss PD, Westcott SL, Ryabin T, Hall JR, Hartmann M, Hollister EB, et al. Introducing

560 mothur: open-source, platform-independent, community-supported software for describing and

561 comparing microbial communities. Appl Environ Microbiol [Internet]. 2009 [cited 2013 Mar

562 1];75:7537-41. Available from: http://aem.asm.org/cgi/content/abstract/75/23/7537

563 12. Bolyen E, Rideout JR, Dillon MR, Bokulich NA, Abnet CC, Al-Ghalith GA, et al.

564 Reproducible, interactive, scalable and extensible microbiome data science using QIIME 2. Nat

565 Biotechnol [Internet]. 2019;37:852-7. Available from: http://www.nature.com/articles/s41587-

$566 \quad 019-0209-9$

567 13. Hildebrand F, Tadeo R, Voigt A, Bork P, Raes J. LotuS: an efficient and user-friendly OTU

568 processing pipeline. Microbiome [Internet]. BioMed Central Ltd; 2014 [cited 2014 Sep 30];2:30.

569 Available from: http://www.microbiomejournal.com/content/2/1/30

570 14. Prodan A, Tremaroli V, Brolin H, Zwinderman AH, Nieuwdorp M, Levin E. Comparing

571 bioinformatic pipelines for microbial 16S rRNA amplicon sequencing. PLoS One. 2020;15.

572 15. Hupfauf S, Etemadi M, Juárez MFD, Gómez-Brandón M, Insam H, Podmirseg SM. CoMA -

573 an intuitive and user-friendly pipeline for amplicon-sequencing data analysis. PLoS One.

$574 \quad 2020 ; 15$.

575 16. Reeder J, Knight R. The "rare biosphere": a reality check. Nat Methods [Internet].

576 2009;6:636-7. Available from: http://www.ncbi.nlm.nih.gov/pubmed/19718016

577 17. Edgar RC. UPARSE: highly accurate OTU sequences from microbial amplicon reads. Nat

578 Methods [Internet]. 2013 [cited 2014 May 23];10:996-8. Available from: 
579 http://www.ncbi.nlm.nih.gov/pubmed/23955772

580 18. Jeon Y-S, Park S-C, Lim J, Chun J, Kim B-S. Improved pipeline for reducing erroneous

581 identification by $16 \mathrm{~S}$ rRNA sequences using the Illumina MiSeq platform. J Microbiol [Internet].

582 2015;53:60-9. Available from: http://link.springer.com/article/10.1007/s12275-015-4601-y\#page-

5831

584 19. Kozich JJ, Westcott SL, Baxter NT, Highlander SK, Schloss PD. Development of a dual-

585 index sequencing strategy and curation pipeline for analyzing amplicon sequence data on the

586 miseq illumina sequencing platform. Appl Environ Microbiol. 2013;79:5112-20.

587 20. Sinclair L, Osman OA, Bertilsson S, Eiler A. Microbial community composition and diversity

588 via 16S rRNA gene amplicons: Evaluating the illumina platform. PLoS One. 2015;10.

589 21. Puente-Sanchez F, Aguirre J, Parro V, Puente-s F, Aguirre J. A novel conceptual approach

590 to read-filtering in high-throughput amplicon sequencing studies. Nucleic Acids Res [Internet].

5912015 [cited 2015 Nov 9];gkv1113-. Available from:

592 http://nar.oxfordjournals.org/content/early/2015/11/06/nar.gkv1113.abstract

593 22. Afgan E, Baker D, Batut B, Van Den Beek M, Bouvier D, Ech M, et al. The Galaxy platform

594 for accessible, reproducible and collaborative biomedical analyses: 2018 update. Nucleic Acids

595 Res. 2018;46.

596 23. Frøslev TG, Kjøller R, Bruun HH, Ejrnæs R, Brunbjerg AK, Pietroni C, et al. Algorithm for

597 post-clustering curation of DNA amplicon data yields reliable biodiversity estimates. Nat

598 Commun [Internet]. Springer US; 2017;8. Available from: http://dx.doi.org/10.1038/s41467-017-

$599 \quad 01312-x$

600 24. McDonald D, Clemente JC, Kuczynski J, Rideout JR, Stombaugh J, Wendel D, et al. The

601 Biological Observation Matrix (BIOM) format or: how I learned to stop worrying and love the

602 ome-ome. Gigascience [Internet]. 2012;1:7. Available from:

603 http://www.pubmedcentral.nih.gov/articlerender.fcgi?artid=3626512\&tool=pmcentrez\&rendertyp $604 \mathrm{e}=\mathrm{abstract}$ 
25. McMurdie PJ, Holmes S. phyloseq: an R package for reproducible interactive analysis and

606 graphics of microbiome census data. Watson M, editor. PLoS One. Public Library of Science;

607 2013;8:e61217.

608 26. Edgar RC. UNOISE2: improved error-correction for Illumina 16S and ITS amplicon

609 sequencing. bioarxiv.

610 27. Fu L, Niu B, Zhu Z, Wu S, Li W. CD-HIT: Accelerated for clustering the next-generation

611 sequencing data. Bioinformatics. 2012;28:3150-2.

612 28. Mahé F, Rognes T, Quince C, de Vargas C, Dunthorn M. Swarm: robust and fast clustering

613 method for amplicon-based studies. PeerJ [Internet]. 2014 [cited 2014 Dec 9];2:e593. Available

614 from: https://peerj.com/articles/593

615 29. Rognes T, Flouri T, Nichols B, Quince C, Mahé F. VSEARCH: A versatile open source tool

616 for metagenomics. PeerJ. 2016;2016:1-22.

617 30. Edgar R. UCHIME2: improved chimera prediction for amplicon sequencing. bioRxiv

618 [Internet]. 2016;074252. Available from: http://biorxiv.org/lookup/doi/10.1101/074252

619 31. Bengtsson-Palme J, Ryberg M, Hartmann M, Branco S, Wang Z, Godhe A, et al. ITSx:

620 Improved software detection and extraction of ITS1 and ITS2 from ribosomal ITS sequences of

621 fungi and other eukaryotes for analysis of environmental sequencing data. Bunce M, editor.

622 Methods Ecol Evol [Internet]. 2013;n/a-n/a. Available from:

623 https://onlinelibrary.wiley.com/doi/10.1111/2041-210X.12073

624 32. Edgar R. UNCROSS2: identification of cross-talk in 16S rRNA OTU tables. 2018;

625 33. Li H. Minimap2: pairwise alignment for nucleotide sequences. Birol I, editor. Bioinformatics

626 [Internet]. 2018;34:3094-100. Available from:

627 https://academic.oup.com/bioinformatics/article/34/18/3094/4994778

628 34. Liu Z, DeSantis TZ, Andersen GL, Knight R. Accurate taxonomy assignments from 16S

629 rRNA sequences produced by highly parallel pyrosequencers. Nucleic Acids Res.

$630 \quad 2008 ; 36: e 120$. 
631 35. Edgar R. SINTAX: a simple non-Bayesian taxonomy classifier for $16 \mathrm{~S}$ and ITS sequences.

632 bioRxiv. 2016;

633 36. Hauswedell H, Singer J, Reinert K. Lambda: the local aligner for massive biological data.

634 Bioinformatics [Internet]. 2014 [cited 2014 Aug 26];30:i349-55. Available from:

635 http://bioinformatics.oxfordjournals.org/cgi/doi/10.1093/bioinformatics/btu439

636 37. Altschul SF, Gish W, Miller W, Myers EW, Lipman DJ. Basic local alignment search tool. J

637 Mol Biol [Internet]. 1990 [cited 2013 Feb 28];215:403-10. Available from:

638 http://dx.doi.org/10.1016/S0022-2836(05)80360-2

639 38. Edgar RC. Search and clustering orders of magnitude faster than BLAST. Bioinforma

640 [Internet]. 2010;26:2460-1. Available from:

641 http://www.ncbi.nlm.nih.gov/entrez/query.fcgi?cmd=Retrieve\&db=PubMed\&dopt=Citation\&list_ui

642 ds=20709691

643 39. Yilmaz P, Parfrey LW, Yarza P, Gerken J, Pruesse E, Quast C, et al. The SILVA and "All-

644 species Living Tree Project (LTP)" taxonomic frameworks. Nucleic Acids Res. 2014;42:D643-8.

645 40. McDonald D, Price MN, Goodrich J, Nawrocki EP, DeSantis TZ, Probst A, et al. An

646 improved Greengenes taxonomy with explicit ranks for ecological and evolutionary analyses of

647 bacteria and archaea. ISME J [Internet]. Nature Publishing Group; 2012 [cited 2013 Sep

648 19];6:610-8. Available from: http://www.nature.com/doifinder/10.1038/ismej.2011.139

649 41. Ritari J, Salojärvi J, Lahti L, de Vos WM. Improved taxonomic assignment of human

650 intestinal 16S rRNA sequences by a dedicated reference database. BMC Genomics [Internet].

651 BMC Genomics; 2015;16:1056. Available from: http://www.biomedcentral.com/1471-

$652 \quad 2164 / 16 / 1056$

653 42. Guillou L, Bachar D, Audic S, Bass D, Berney C, Bittner L, et al. The Protist Ribosomal

654 Reference database (PR2): A catalog of unicellular eukaryote Small Sub-Unit rRNA sequences

655 with curated taxonomy. Nucleic Acids Res. 2013;41.

656 43. Jones JC, Fruciano C, Hildebrand F, Al Toufalilia H, J Balfour N, Bork P, et al. Gut 
657 microbiota composition is associated with environmental landscape in honey bees. Ecol Evol

658 [Internet]. 2017;1-11. Available from: http://doi.wiley.com/10.1002/ece3.3597

659 44. Kõljalg U, Nilsson RH, Abarenkov K, Tedersoo L, Taylor AFS, Bahram M, et al. Towards a

660 unified paradigm for sequence-based identification of fungi. Mol Ecol [Internet]. 2013;22:5271-

661 7. Available from: http://doi.wiley.com/10.1111/mec.12481

662 45. Katoh K, Standley DM. MAFFT Multiple Sequence Alignment Software Version 7:

663 Improvements in Performance and Usability. Mol Biol Evol [Internet]. 2013;30:772-80. Available

664 from: http://www.ncbi.nlm.nih.gov/pubmed/23329690

665 46. Sievers F, Wilm A, Dineen D, Gibson TJ, Karplus K, Li W, et al. Fast, scalable generation of

666 high-quality protein multiple sequence alignments using Clustal Omega. Mol Syst Biol [Internet].

6672011 [cited 2013 Sep 19];7:539. Available from: http://www.ncbi.nlm.nih.gov/pubmed/21988835

668 47. Price MN, Dehal PS, Arkin AP. FastTree 2--approximately maximum-likelihood trees for

669 large alignments. Poon AFY, editor. PLoS One [Internet]. Public Library of Science; 2010 [cited

6702013 Oct 30];5:e9490. Available from: http://dx.plos.org/10.1371/journal.pone.0009490

671 48. Nguyen L-T, Schmidt HA, von Haeseler A, Minh BQ. IQ-TREE: A Fast and Effective

672 Stochastic Algorithm for Estimating Maximum-Likelihood Phylogenies. Mol Biol Evol [Internet].

673 2015;32:268-74. Available from: https://academic.oup.com/mbe/article-

674 lookup/doi/10.1093/molbev/msu300

675 49. Amir A, McDonald D, Navas-Molina JA, Kopylova E, Morton JT, Zech Xu Z, et al. Deblur

676 Rapidly Resolves Single-Nucleotide Community Sequence Patterns. Gilbert JA, editor.

677 mSystems [Internet]. 2017;2:1-7. Available from:

678 https://journals.asm.org/doi/10.1128/mSystems.00191-16

679 50. Rivers AR, Weber KC, Gardner TG, Liu S, Armstrong SD. ITSxpress: Software to rapidly

680 trim internally transcribed spacer sequences with quality scores for marker gene analysis

681 [version 1; peer review: 2 approved]. F1000Research. 2018;7.

682 51. Nilsson RH, Anslan S, Bahram M, Wurzbacher C, Baldrian P, Tedersoo L. Mycobiome 
683 diversity: high-throughput sequencing and identification of fungi. Nat. Rev. Microbiol. 2019.

684 52. Bokulich NA, Rideout JR, Mercurio WG, Shiffer A, Wolfe B, Maurice CF, et al. mockrobiota:

685 a Public Resource for Microbiome Bioinformatics Benchmarking. mSystems. 2016;1.

686 53. Saary P, Forslund K, Bork P, Hildebrand F. RTK: efficient rarefaction analysis of large

687 datasets. Birol I, editor. Bioinformatics [Internet]. 2017;33:2594-5. Available from:

688 https://academic.oup.com/bioinformatics/article/33/16/2594/3111845

689 54. Bokulich NA, Kaehler BD, Rideout JR, Dillon M, Bolyen E, Knight R, et al. Optimizing

690 taxonomic classification of marker-gene amplicon sequences with QIIME 2's q2-feature-

691 classifier plugin. Microbiome. 2018;6.

692 55. Větrovský T, Baldrian P. The Variability of the $16 \mathrm{~S}$ rRNA Gene in Bacterial Genomes and Its

693 Consequences for Bacterial Community Analyses. Neufeld J, editor. PLoS One [Internet]. Public

694 Library of Science; 2013 [cited 2013 Mar 2];8:e57923. Available from:

695 http://dx.plos.org/10.1371/journal.pone.0057923

696 56. Kunin V, Engelbrektson A, Ochman H, Hugenholtz P. Wrinkles in the rare biosphere:

697 pyrosequencing errors can lead to artificial inflation of diversity estimates. Environ Microbiol

698 [Internet]. 2010 [cited 2013 May 21];12:118-23. Available from:

699 http://www.ncbi.nlm.nih.gov/pubmed/19725865 


\section{Figures:}

714

a)

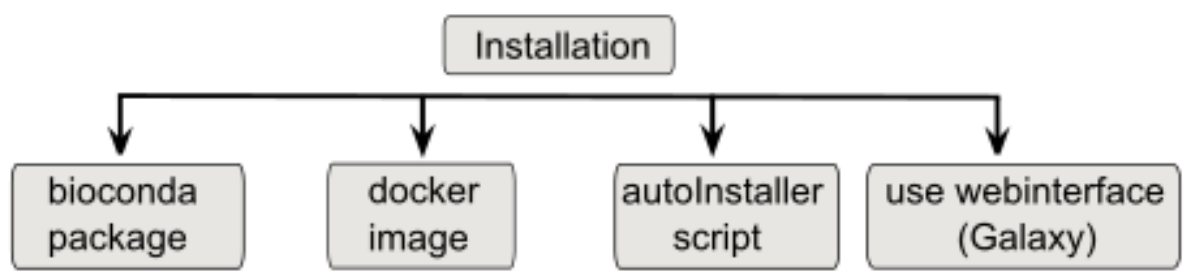

b)

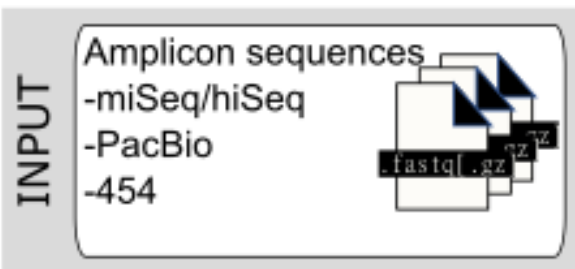

Sample ID,

[either] -file name

-read header tag

-demultiplex dual/single Barcode

[optional] primers, illumina run, other metadata

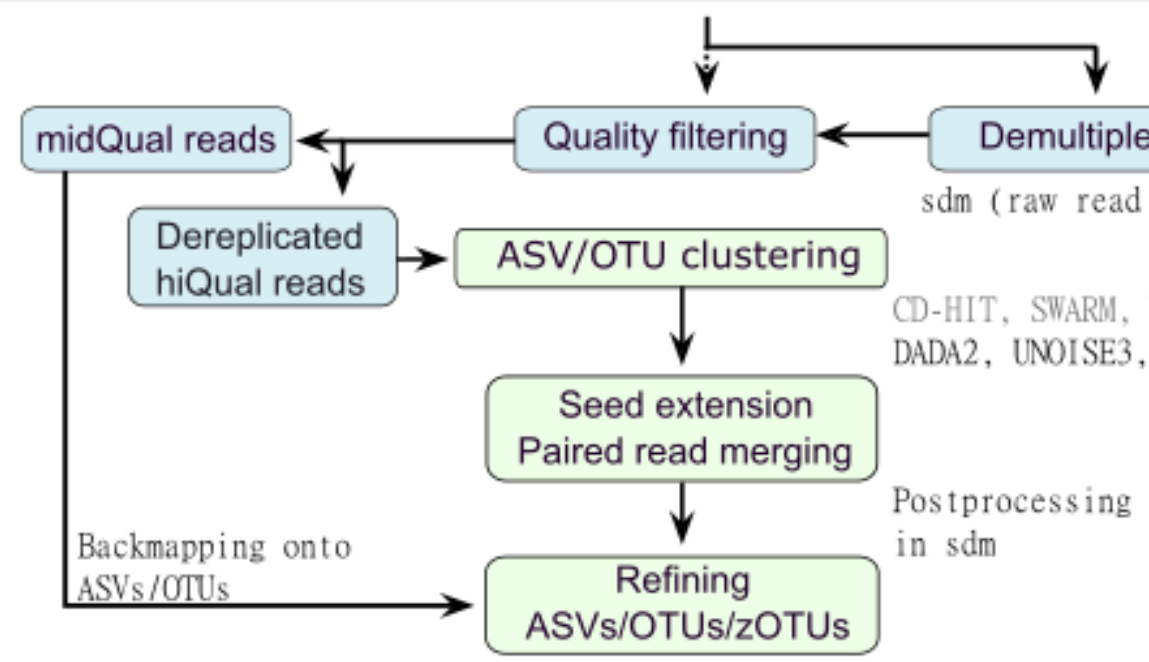

Offtarget removal, UCHIME3 (de novo/ref)

ASV re-clustering

ITSX, LULU, UNCROSS2

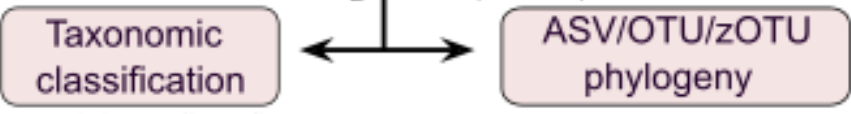

LCA (BLAST, Lambda, USEARCH, CLUSTAL OMEGA, FastTree2, VSEARCH), RDP-classifier, SINTAX MAFFT, IQ-TREE2

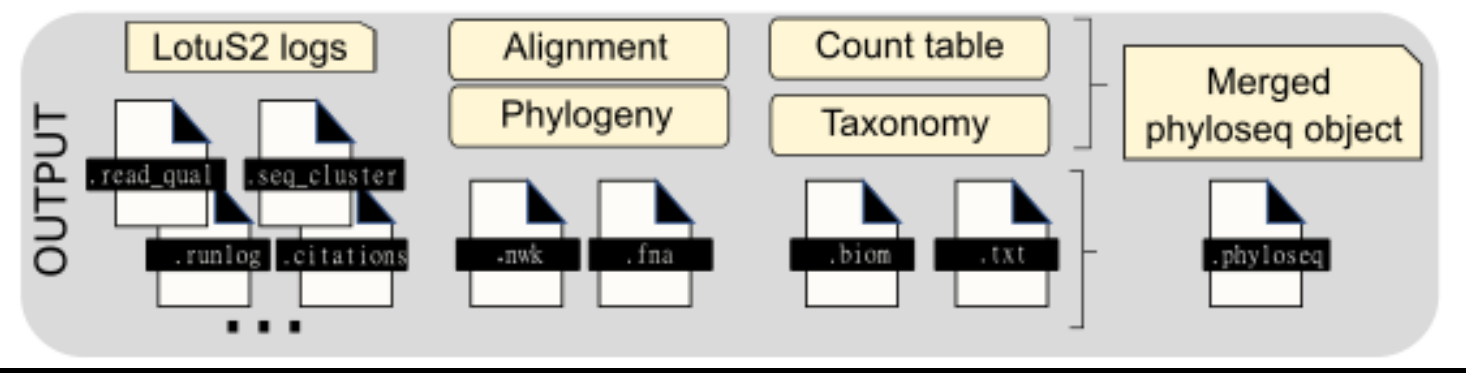


718 a) LotuS2 can be installed either through i) Bioconda, ii) GitHub with the provided autolnstaller 719 script or iii) using a Docker image. Alternatively, iv) Galaxy web servers can also run LotuS2

720 (e.g. https://usegalaxy.eu/) b) LotuS2 accepts amplicon reads from different sequencing

721 platforms, along with a map file that describes barcodes, file locations, sample IDs and other

722 information. After demultiplexing and quality filtering, high-quality reads are clustered into either

723 ASVs or OTUs. The optimal sequence representing each OTU/ASV is calculated in the seed

724 extension step, where read pairs are also merged. Mid-quality reads are subsequently mapped

725 onto these sequence clusters, to increase cluster representation in abundance matrices. From

726 OTU/ASV sequences, a phylogenetic tree is constructed, and each cluster is taxonomically

727 assigned. These results are made available in multiple standard formats, such as tab-delimited

728 files, .biom or phyloseq objects, to enable downstream analysis. New options in LotuS2 for each

729 step are denoted with black colour whereas options in grey font were already available in LotuS. 
A
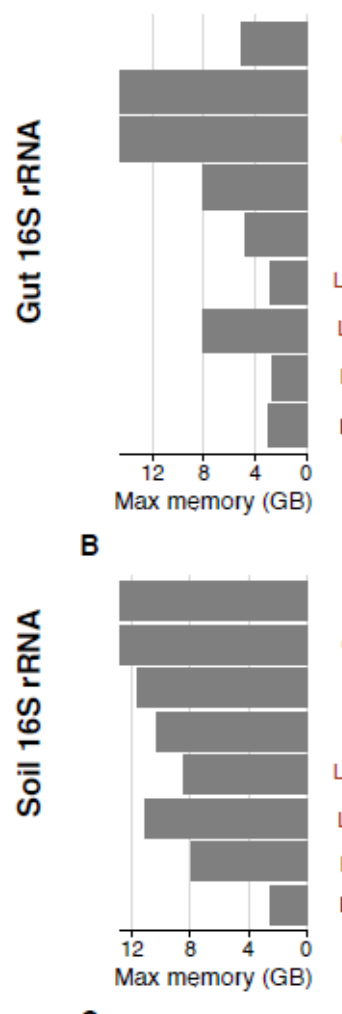

c

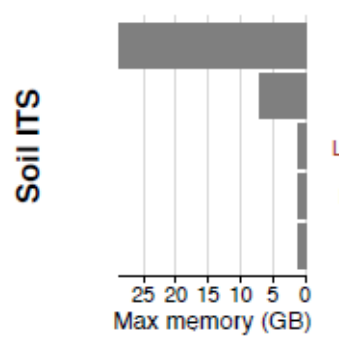

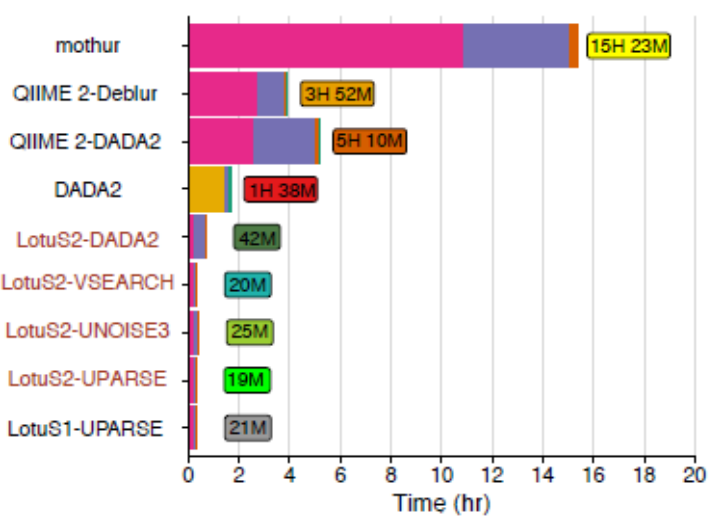
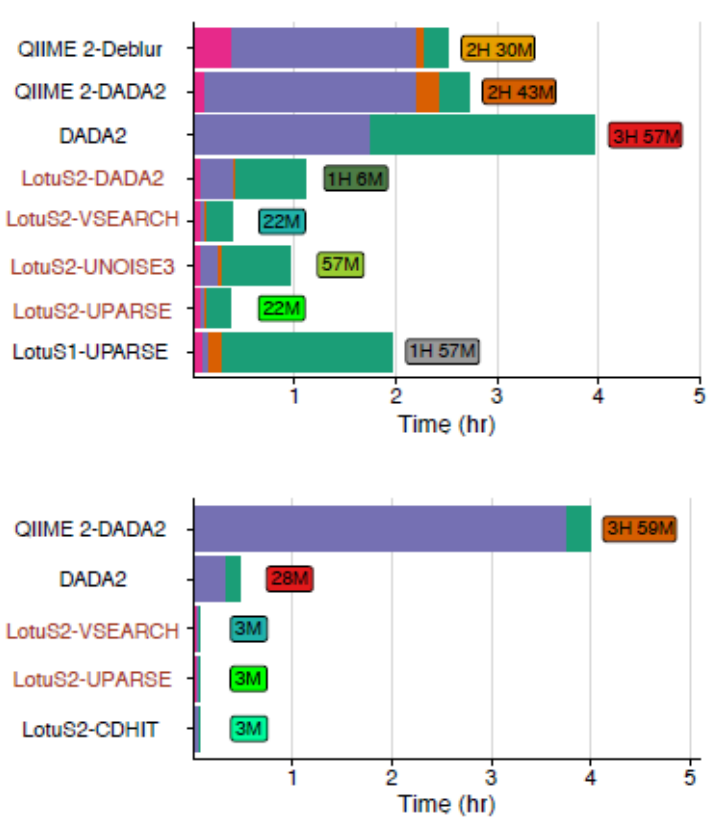

D
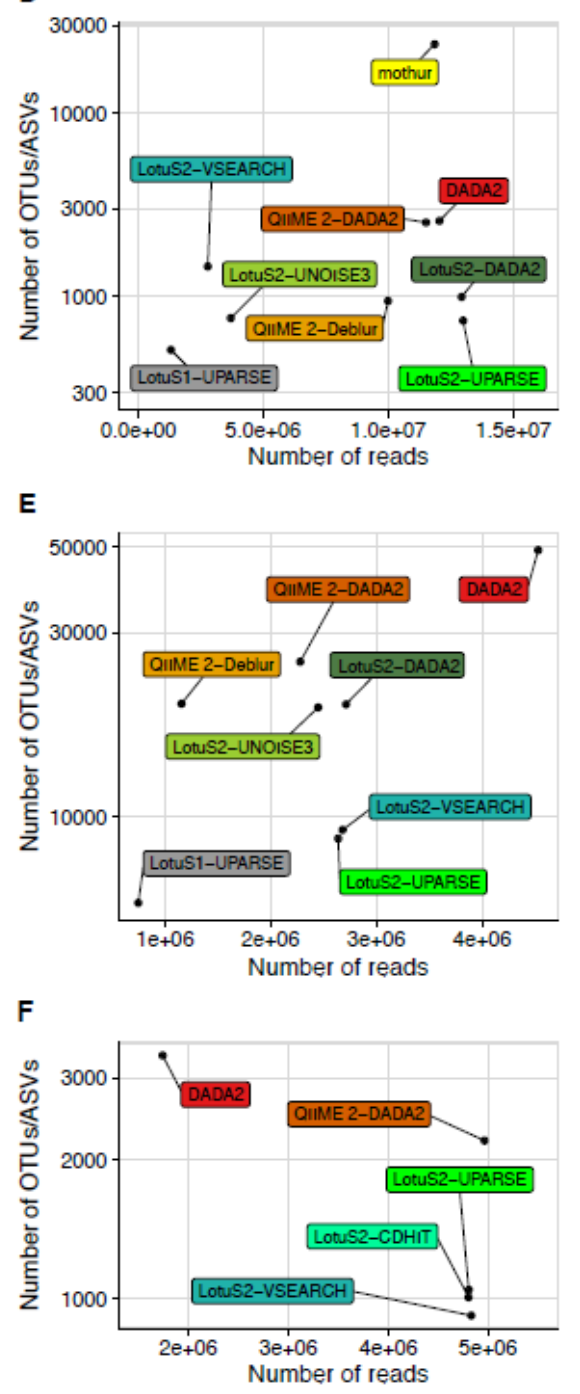

\section{Figure 2: Computational performance of amplicon sequencing pipelines} processed to benchmark resource usage of each pipeline, run on the same system under equal conditions (12 cores, max 150Gb memory). In all pipelines, OTUs/ASVs were classified by similarity comparisons to SILVA 138.1. In LotuS2, LAMBDA was used to align sequences for all clustering algorithms.

740 Pipeline runs were separated by common steps (pre-processing, sequence clustering, taxonomic classification and phylogenetic tree construction and/or off-target removal). Because native DADA2 cannot demultiplex reads, we used the average demultiplexing time of QIIME 2 and LotuS2 (LotuS2 demultiplexed, unfiltered reads were provided to DADA2). LotuS2 pipelines are labelled with red colour. 

clusters (OTUs or ASVs) to retrieved read counts in the final output matrix of each pipeline. Note that mothur results on soil-16S are not shown, because the pipeline rejected with default parameters all sequences.

A

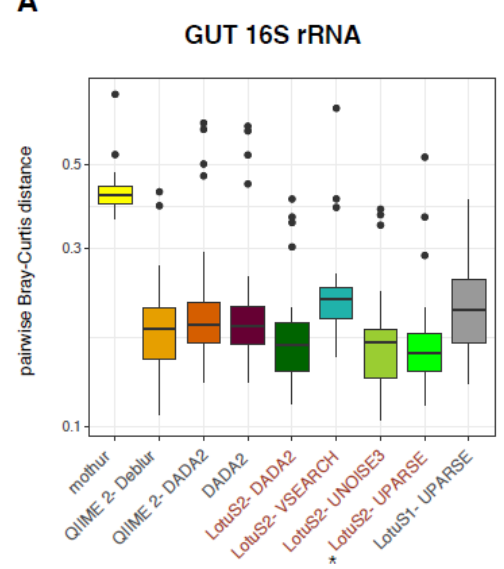

D

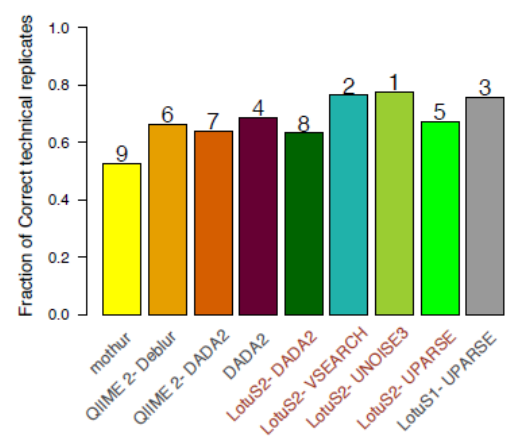

B

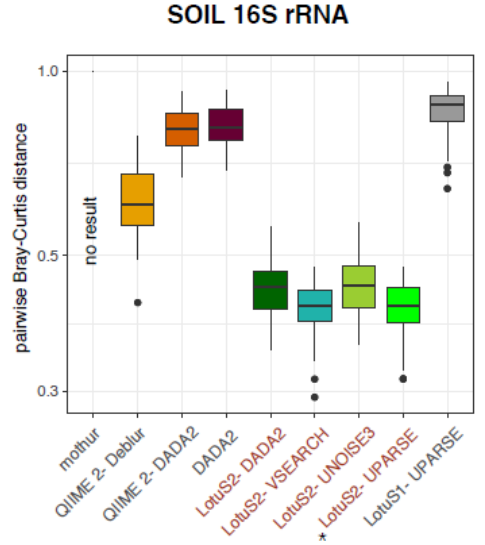

E

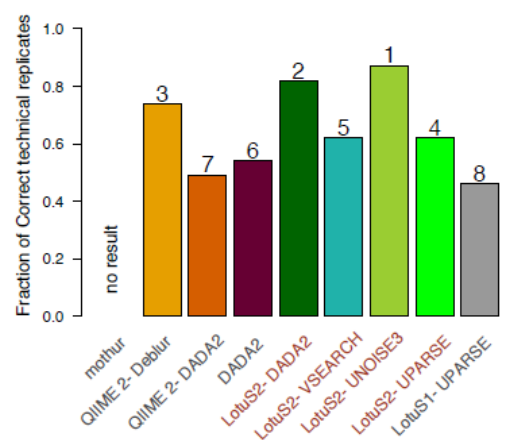

C

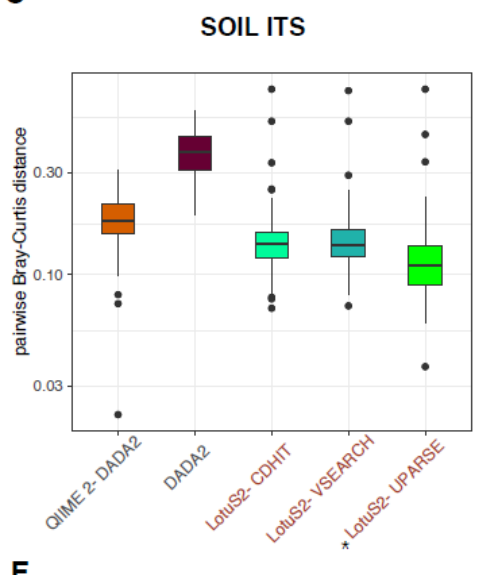

F

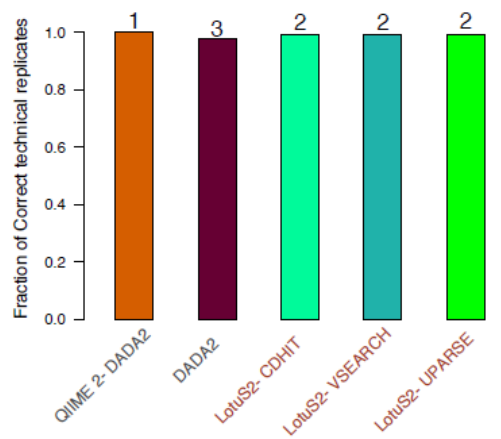

Figure 3- Reproducibility from different amplicons sequence data analysis pipelines.

753

754

755

756

757

758

759

760

761

762

763

764

Three independent datasets were used to represent different biomes and amplicon technologies, using $A, D$ ) human faecal samples (16S rRNA gene, $N=40$ replicates). $B, E$ ) soil samples (16S rRNA gene, $\mathrm{N}=50$ replicates) and $\mathrm{C}, \mathrm{F}$ ) soil samples (ITS 2, $\mathrm{N}=50$ replicates). A-C) Bray-Curtis distances among technical replicate samples are used to assess the reproducibility of community compositions by different pipelines. The pipeline with the lowest $\mathrm{BCd}$ in each subfigure is denoted with a star $\left(^{*}\right)$. The significance of pairwise comparisons of each pipeline is calculated using the Tukey's HSD test (Supp. Table 2).

D-F) Further, the fraction of technical replicates being closest to each other (BCd) was calculated to simulate identifying technical replicates without additional knowledge. Numbers above bars are the ordered pipelines performing best.

Lower Bray-Curtis distances between technical replicates and a higher fraction of correct technical replicates indicate better reproducibility. LotuS2 pipelines are labelled with red colour. 
A Fraction of TP reads

Precision

Recall F-score

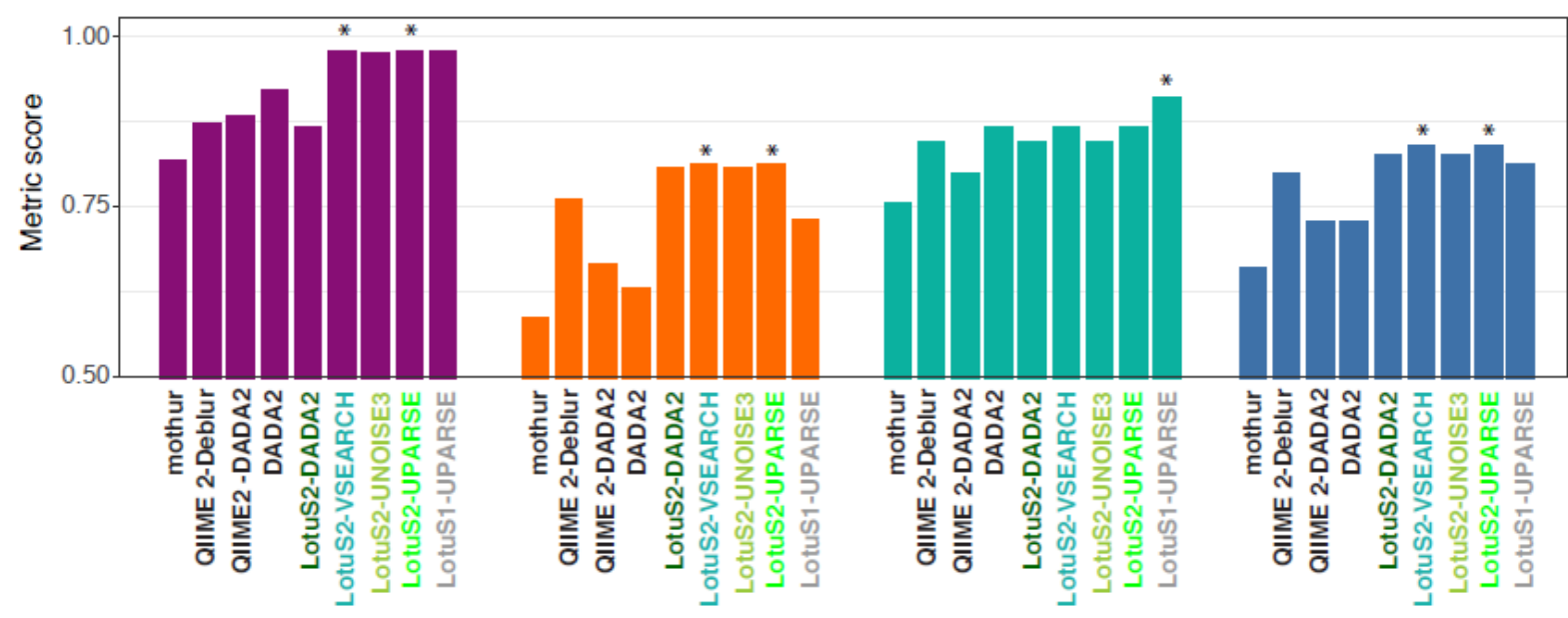

B

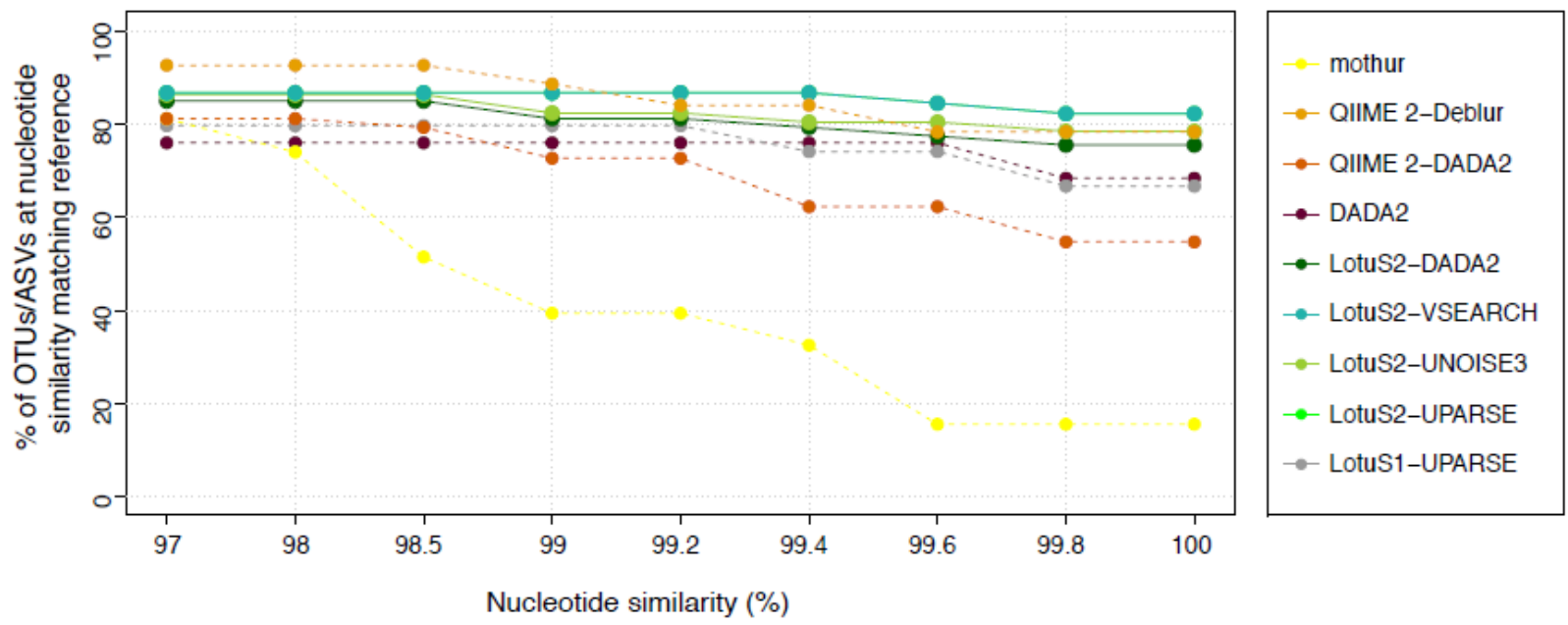

Nucleotide similarity (\%)

Figure 4- Benchmarking of amplicon sequence data analysis pipeline's performance using a mock community with known species composition

770 A) Accuracy of each pipeline in predicting the mock community composition at genus level. For benchmarking we compared the fraction of reads assigned to true genera and both correctly and erroneously recovered genera. Precision, Recall and F-score were calculated based on the true positive, false positive and false negative taxa identified. At species level, LotuS2 excelled as well in these statistics (Supp. Figure 9).

B) Percentage of true positive ASVs/OTUs having a nucleotide identity $\geq$ indicated thresholds to 16S rRNA gene sequences of genomes from the mock community.

777 Pipeline(s) showing the highest performance in each comparison is denoted with a star $\left(^{*}\right)$. TP, 778 true positive; ASV, amplicon sequencing variant; OTU, operational taxonomic unit. 
bioRxiv preprint doi: $h t t p s: / / d o i . o r g / 10.1101 / 2021.12 .24 .474111$; this version posted December 24,2021 . The copyright holder for this preprint (which was not certified by peer review) is the author/funder, who has granted bioRxiv a license to display the preprint in perpetuity. It is made available under aCC-BY-NC-ND 4.0 International license.

\section{Supp. Figures and Tables:}

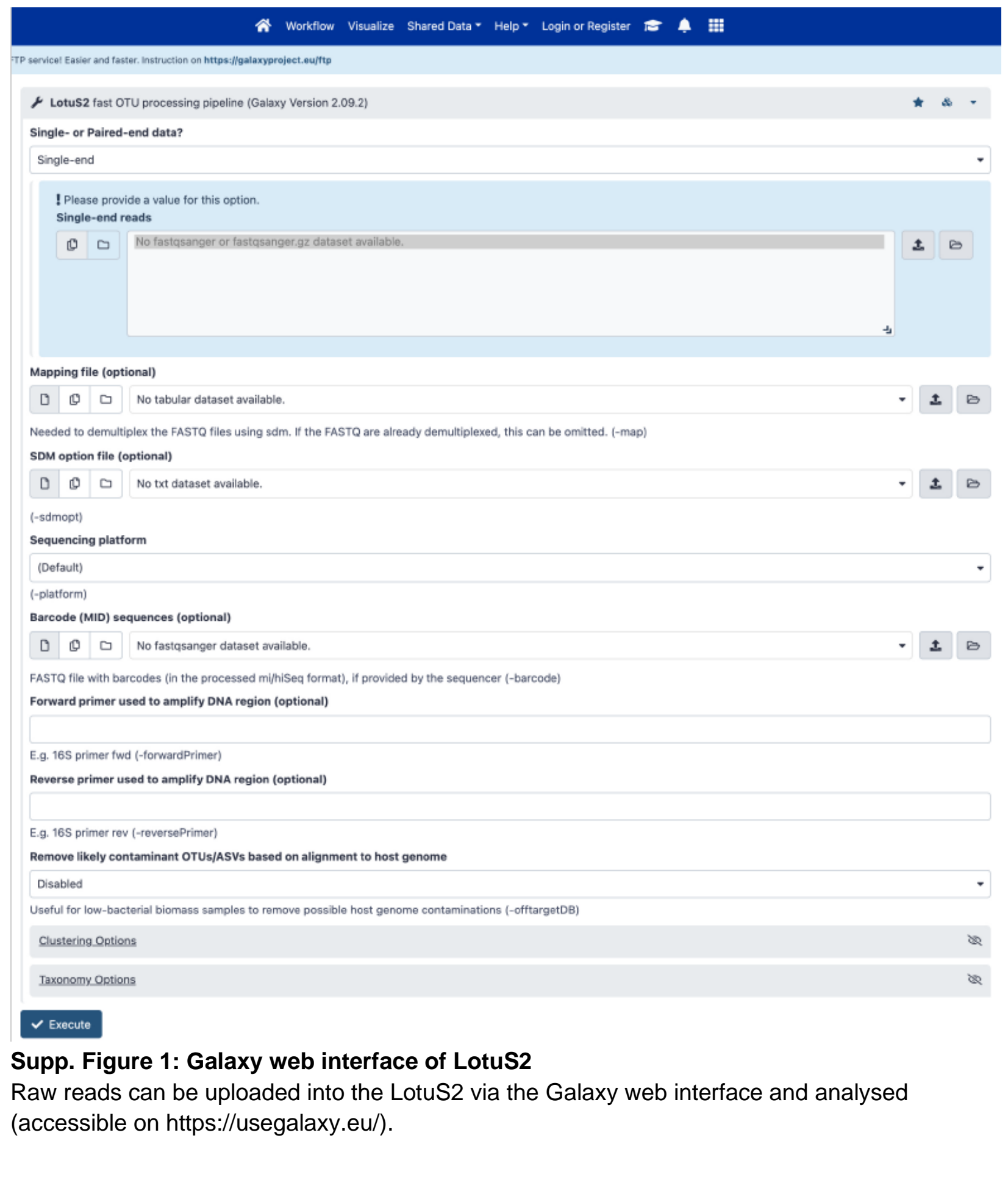


A

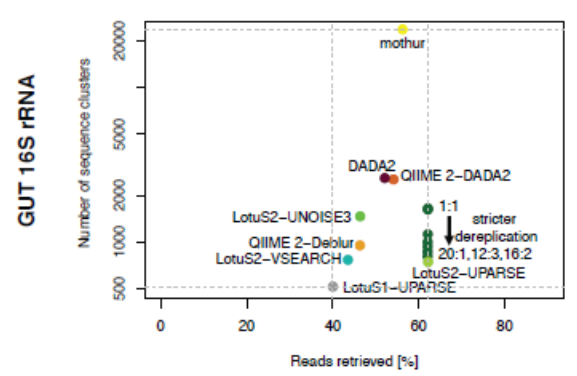

D

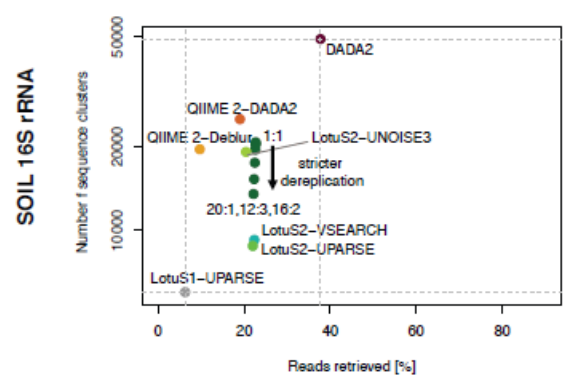

G

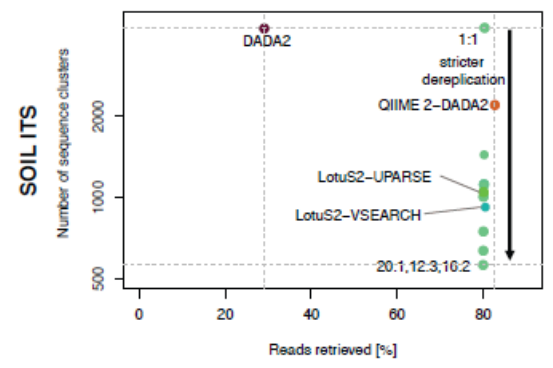

B
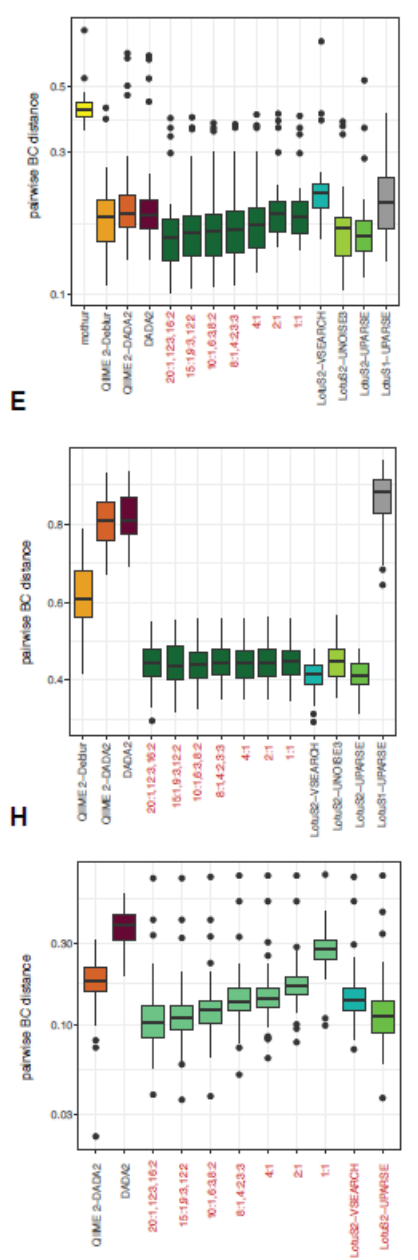

C

F
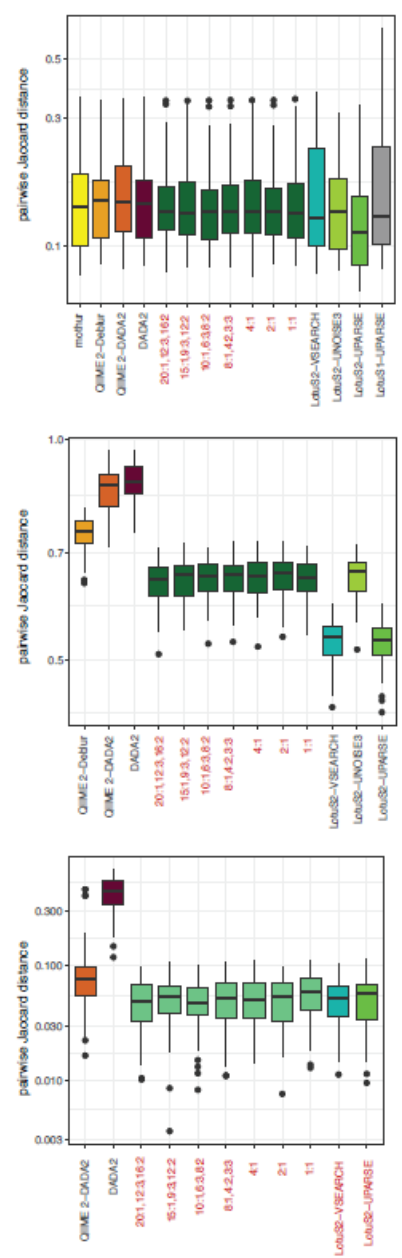

788

789

790

791

792

793

794

795

796

797

798

799

800

Supp. Figure 2- Reproducibility and data usage efficiency respective to dereplication filtering.

A, D and G) Data usage efficiency of each tested pipeline at different dereplication parameters of LotuS2 (from strictest to least strict dereplication: 20:1,12:3,6:2; 15:1,9:3,12:2; 10:1,6:3,8:2;

8:1,4:2,3:3 (default); $4: 1 ; 2: 1$ and 1:1) using DADA2 or CD-HIT clustering for $16 \mathrm{~S}$ and ITS dataset, respectively, by comparing the number of sequence clusters (OTUs/ASVs) to retrieved read counts in final output matrix.

The dereplication can be fine controlled through a syntax. For example, 8:1,4:2,3:3 means that a read is accepted, if it occurs $>=8$ times in $>=1$ samples or $>4$ times total in $>=2$ samples or $>=3$ times in $>=3$ samples. 
A

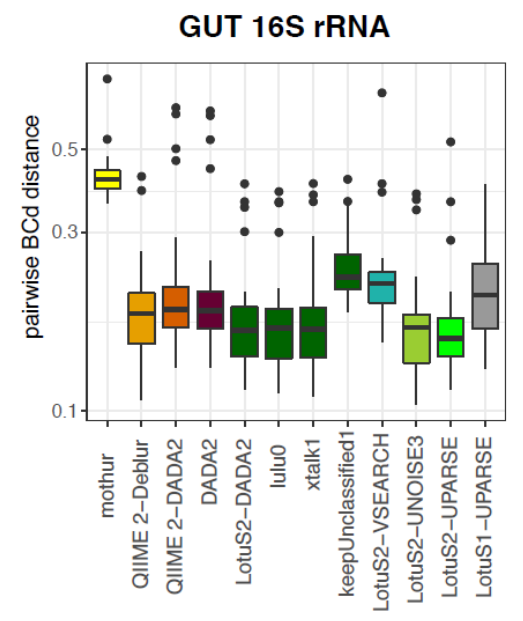

B

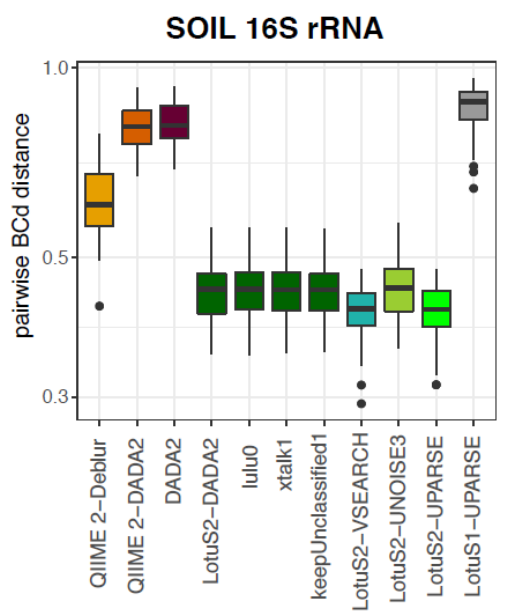

C

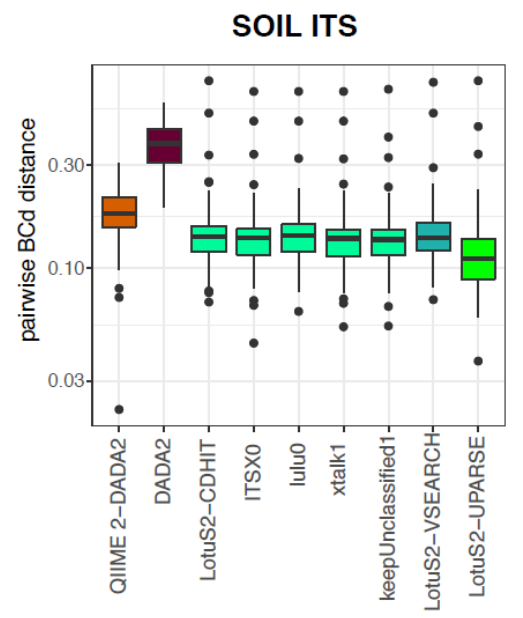

Supp. Figure 3- Reproducibility of the technical replicates respective to different LotuS2 non-default parameters

Bray-Curtis distances between technical replicates of A) gut-16S B) soil-16S and C) soil-ITS datasets using default and non-default parameters (LotuS2 flags: -lulu 0, -xtalk 1, keepUnclassified 1, -ITSx 0, where 1 means the option is activated; 0 means deactivated). When activated, -lulu option uses LULU R package [23] to merge OTUs/ASVs based on their co-occurrences; -xtalk option checks for cross-talk [32], -keepUnclassified includes unclassified (i.e. not matching to any taxon in the taxonomy database) OTUs/ASVs in the final matrix and ITSx activates the ITSx program [31] to only retain OTUs fitting to ITS1/ITS2 $\mathrm{hmm}$ models. 
A

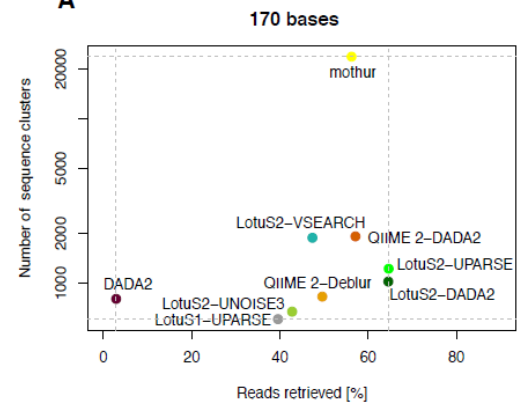

D

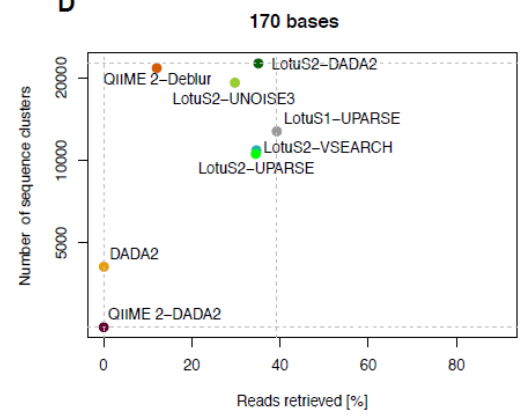

B

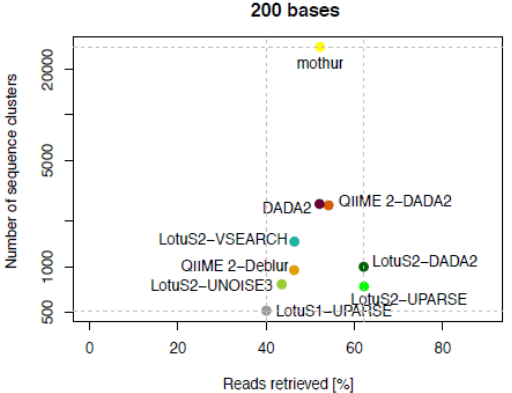

E

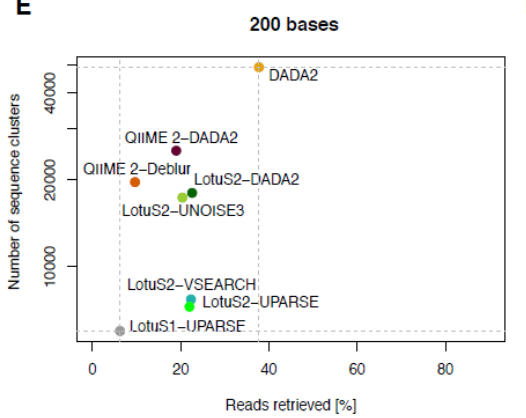

C

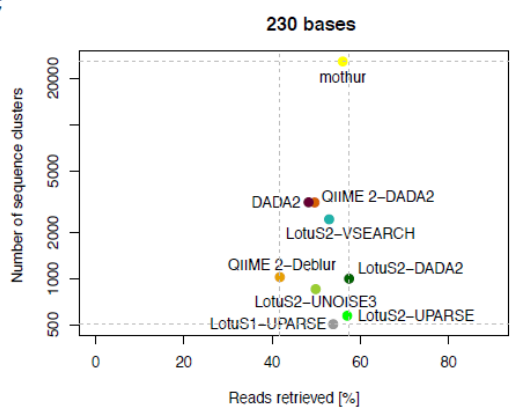

$\mathbf{F}$

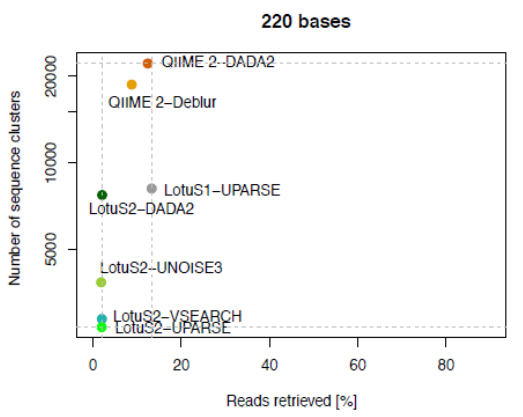

815 Supp. Figure 4- Data usage efficiency of different amplicon sequence data analysis

816 pipelines.

818 Data usage efficiency on gut 16S rRNA (gut-16S), soil 16S rRNA (soil-16S) and Soil ITS (soil819 ITS) amplicons, tested with different pipelines at different read truncation lengths (170, 200, 230 $820 \& 170,200,220$ bases for the gut and soil datasets, respectively), by comparing the number of 821 sequence clusters (ASVs /OTUs) to retrieved read counts in the final output matrix of each 822 pipeline. In all other analysis, default values were used for LotuS2 (200 bases). 
A

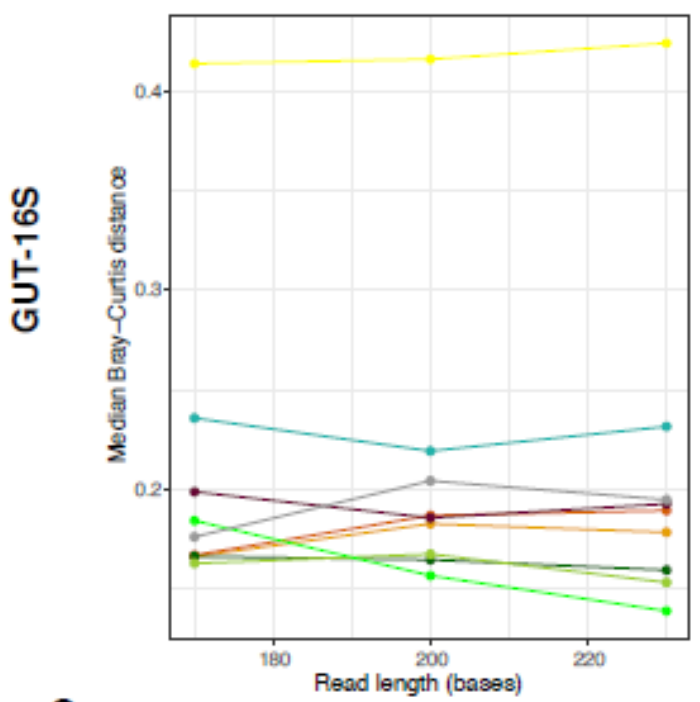

C

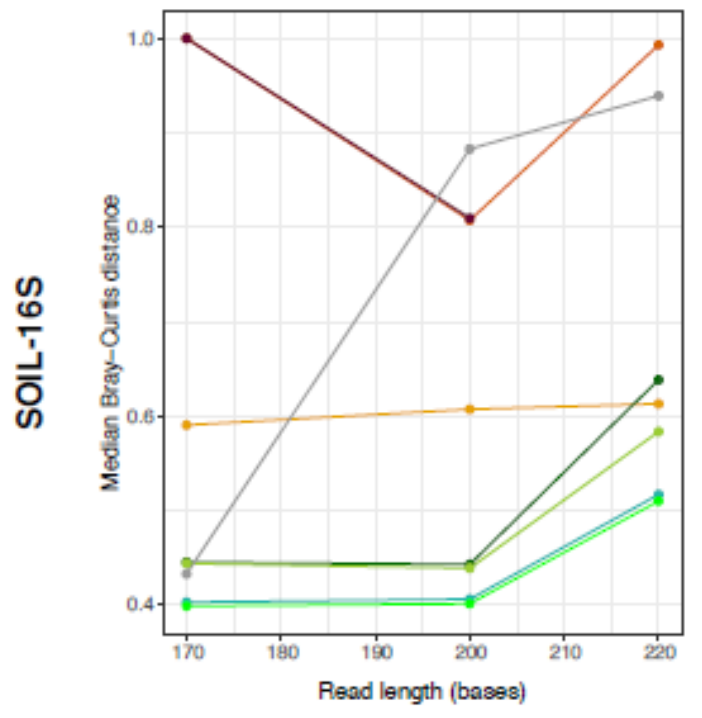

B

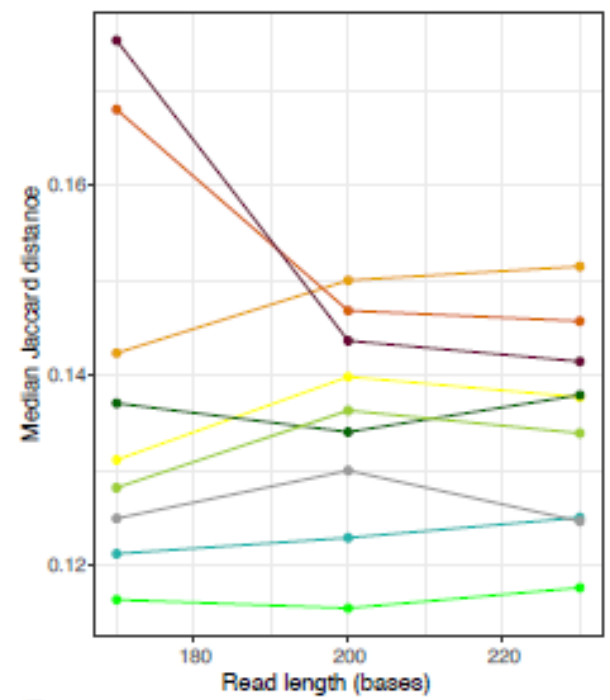

D

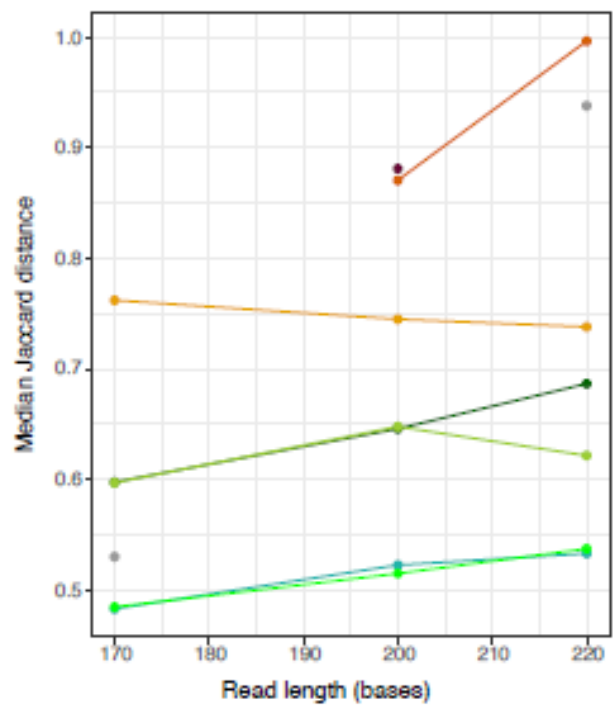

-o- mothur

$\rightarrow$ QIIME 2-Debiur

$\rightarrow$ QIMME 2-DADA2

$\rightarrow$ DADA2

$\rightarrow$ LOtUS2-DADA2

$\rightarrow-$ LOLUS2-VSEARCH

$\rightarrow$ LOtuS2-UNOISE3

$\rightarrow-$ LOLLS2-UPARSE

$\because$ LOtUS1-UPARSE

\section{Supp. Figure 5- Reproducibility of beta diversity at different read truncation lengths}

827 Reproducibility of sequenced technical replicates, by measuring the Bray-Curtis ( $A$ and $C$ ) and Jaccard distances $(B$ and $D)$ of the microbiome composition among technical replicate samples. Two datasets were used to represent different biomes and amplicon technologies, using (A, B) and human faecal samples (16S rRNA primer, $\mathrm{N}=40$ replicates) and (C, D) soil samples (16S rRNA, V4-V5 region primers, $\mathrm{N}=50$ replicates). Lower Bray-Curtis or Jaccard distances between technical replicates indicate better reproducibility of community compositions. Default pipeline parameters and recommended settings for each dataset were used (Please see the Supp. Text for further information). 
A

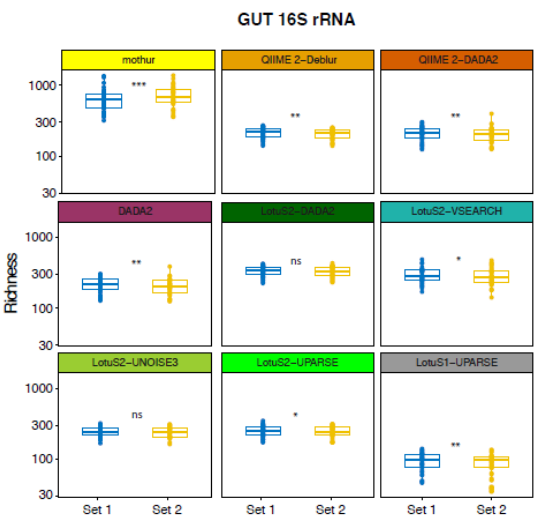

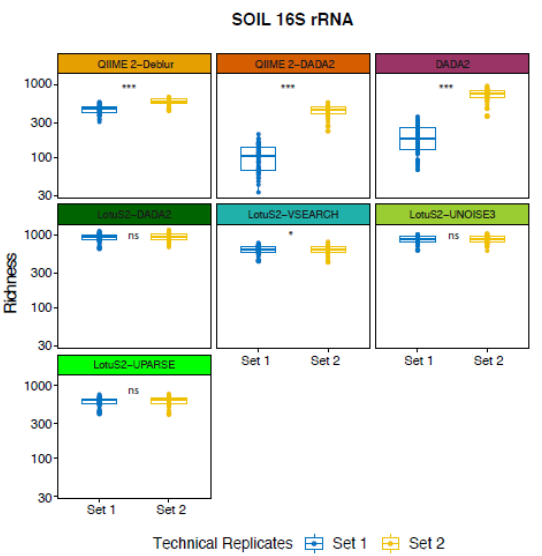

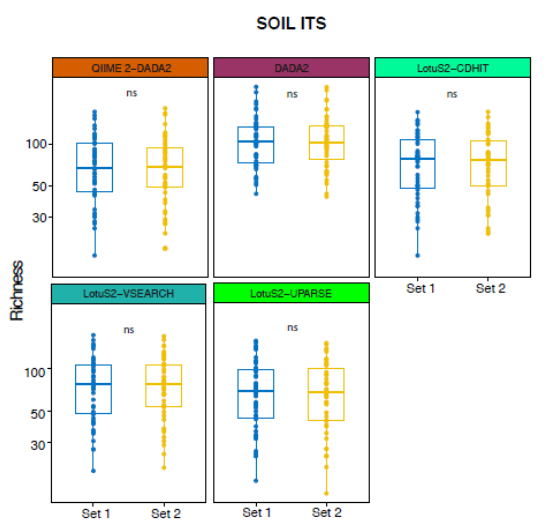

A

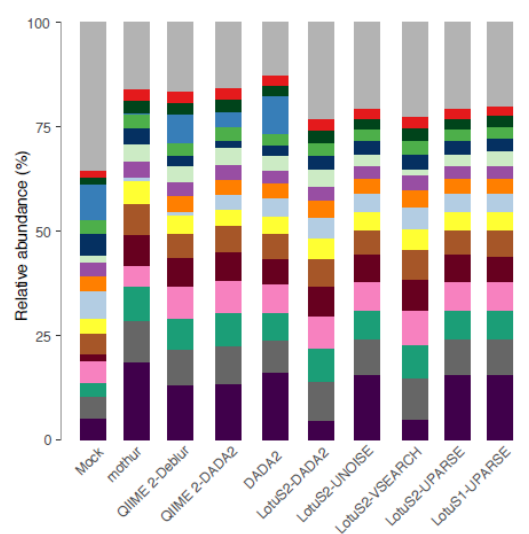
significant), respectively).

Supp. Figure 6: Reproducibility of alpha diversity between technical replicates.

OTU/ASV Richness was calculated for A) gut-16S B) soil-16S and C) soil-ITS datasets. Samples were rarefied to an equal number of reads per sample before calculating richness, and any samples whose replicate pair was removed after rarefaction (because of having lower number of reads than the rarefaction depth) were excluded from further analysis. LotuS1 results for soil-16S were removed due to too many samples being removed in rarefactions. Significance of differences in richness between the sets were calculated based on the paired samples Wilcoxon test $\left({ }^{* * *},{ }^{* *},{ }^{*}\right.$ and "ns" denotes $p<0.0005, p<0.005, p<0.05$ and $p>0.05$ (i.e. not

851 Supp. Figure 7: Observed composition of the mock community compared to the 852 composition predicted by each pipeline

853 A) Relative abundances of the 16 orders having the highest abundance.

854 B) Bray-Curtis distance based PCoA of the observed composition of the mock sample and 855 composition predicted by each pipeline 
A

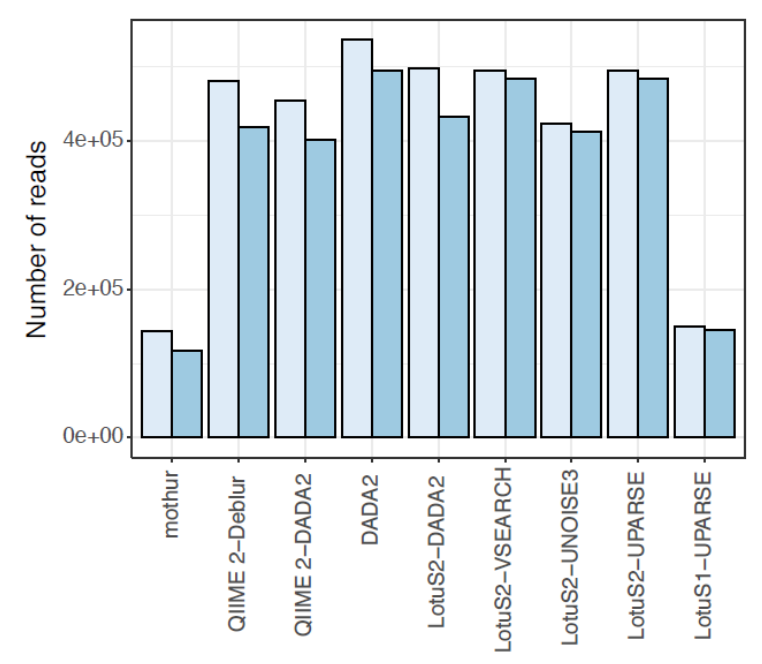

Total number of reads

Number of reads assigned to true taxa
B

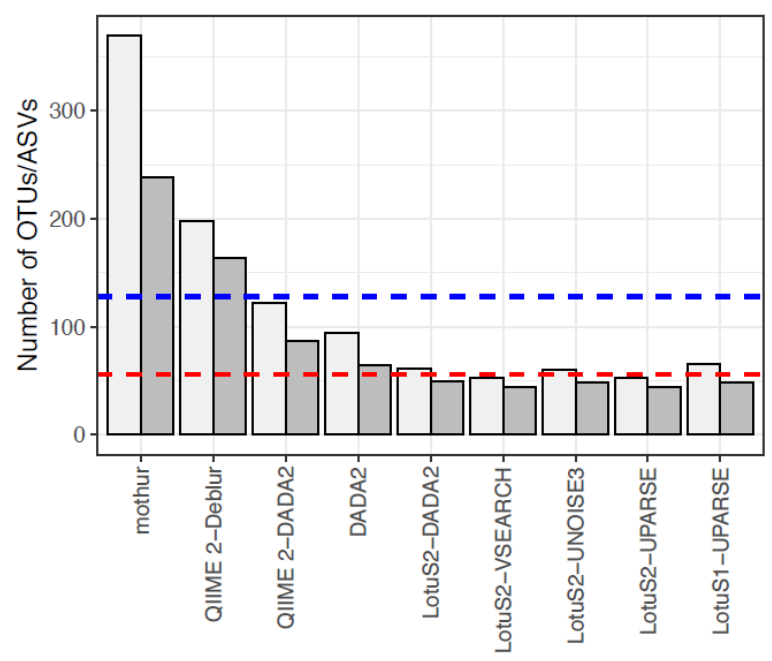

Number of OTUs/ASVs

Number of OTUs/ASVs assigned to true taxa

\section{Supp. Figure 8: Number of reads and OTUs/ASVs and those assigned true taxa at genus}

858 level by each pipeline in the analysis of the mock community

859 Total number of $A$ ) reads retrieved by each pipeline and those assigned to true taxa at genus level B) OTUs/ASVs generated by each pipeline and those assigned to true taxa at genus level. Blue and red line indicates number of $16 \mathrm{~S}$ gene copies and species, respectively, in the mock community.

863

864 


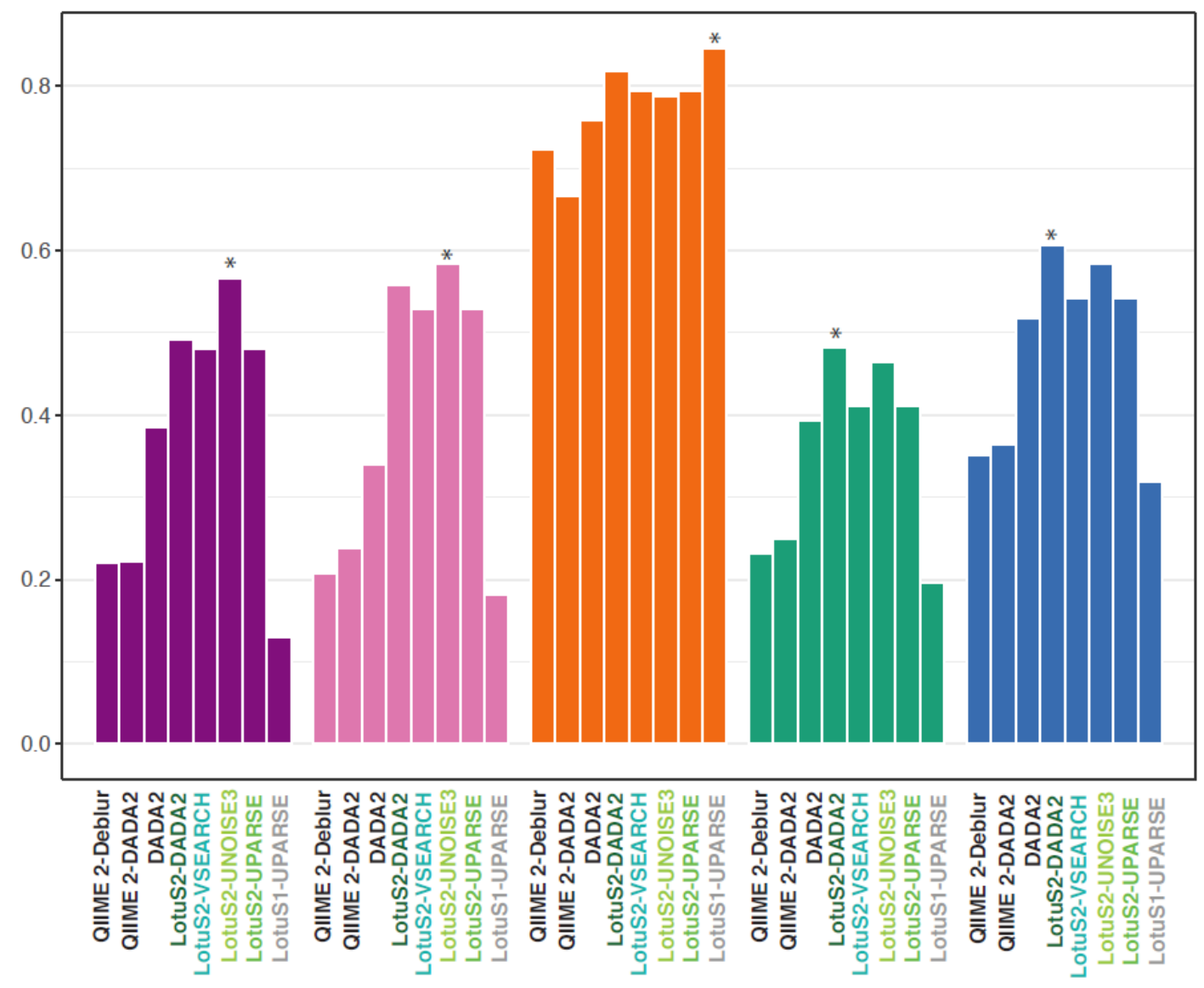

\section{Supp. Figure 9: Accuracy of each pipeline in predicting the mock community} composition at species level. 


\begin{tabular}{lcc}
\hline \multicolumn{3}{c}{ Gut-16S } \\
\hline & Number of reads & Number of OTUs/ASVs \\
mothur & 11855762 & 23736 \\
QIIME 2-Deblur & 9995254 & 950 \\
QIIME 2-DADA2 & 11510552 & 2539 \\
DADA2 & 12048048 & 2591 \\
LotuS2-DADA2 & 12935664 & 999 \\
LotuS2-UNOISE3 & 3698064 & 766 \\
LotuS2-UPARSE & 12995784 & 742 \\
LotuS2-VSEARCH & 2778696 & 1464 \\
LotuS1-UPARSE & 1305288 & 514
\end{tabular}

\begin{tabular}{lcc}
\hline \multicolumn{3}{c}{ Soil-16S } \\
\hline & Number of reads & Number of OTUs/ASVs \\
QIIME 2-Deblur & 1157357 & 19641 \\
QIIME 2-DADA2 & 2278731 & 25229 \\
DADA2 & 4526920 & 49111 \\
LotuS2-DADA2 & 2710629 & 19568 \\
LotuS2-UNOISE3 & 2448475 & 19217 \\
LotuS2-UPARSE & 2637572 & 8789 \\
LotuS2-VSEARCH & 2678716 & 9250 \\
LotuS1-UPARSE & 749449 & 5987
\end{tabular}

\begin{tabular}{lcc}
\hline & Soil-ITS \\
\hline & Number of reads & Number of OTUs/ASVs \\
QIIME 2-DADA2 & 4962260 & 2203 \\
DADA2 & 1742895 & 3368 \\
LotuS2-UPARSE & 4805387 & 1046 \\
LotuS2-VSEARCH & 4829288 & 920 \\
LotuS2-CDHIT & 2678716 & 1008
\end{tabular}

884 Supp. Table 1: Read counts and number of OTUs/ASVs in the OTU/ASV matrix of each 885 pipeline.

Supp. Table 2: Significance of differences between each pipeline in the reproducibility of beta diversity between the technical replicates on the Tukey's HSD test. 


\begin{tabular}{lcc}
\hline & \multicolumn{2}{c}{ Spearman Correlation } \\
\hline & p.value & correlation coefficient \\
mothur & $1.83 \mathrm{E}-07$ & 0.544018417 \\
QIIME 2-Deblur & $1.57 \mathrm{E}-15$ & 0.747912391 \\
QIIME 2-DADA2 & $3.76 \mathrm{E}-12$ & 0.680648974 \\
DADA2 & $6.77 \mathrm{E}-12$ & 0.674725632 \\
LotuS2-DADA2 & $3.26 \mathrm{E}-12$ & 0.682064113 \\
LotuS2-VSEARCH & $2.80 \mathrm{E}-17$ & 0.776030912 \\
LotuS2-UNOISE3 & $4.99 \mathrm{E}-14$ & 0.720369663 \\
LotuS2-UPARSE & $2.80 \mathrm{E}-17$ & 0.776030912 \\
LotuS2-UPARSE & $1.32 \mathrm{E}-19$ & 0.808037907
\end{tabular}

\begin{tabular}{lcc}
\hline & Pearson Correlation \\
\hline & p.value & correlation coefficient \\
mothur & $3.99 \mathrm{E}-07$ & 0.531185654 \\
QIIME 2-Deblur & $1.99 \mathrm{E}-11$ & 0.663501229 \\
QIIME 2-DADA2 & $3.91 \mathrm{E}-09$ & 0.600486282 \\
DADA2 & $7.72 \mathrm{E}-12$ & 0.673389135 \\
LotuS2-DADA2 & $6.62 \mathrm{E}-05$ & 0.43083946 \\
LotuS2-VSEARCH & $2.68 \mathrm{E}-09$ & 0.605505625 \\
LotuS2-UNOISE3 & $1.22 \mathrm{E}-08$ & 0.584843731 \\
LotuS2-UPARSE & $2.68 \mathrm{E}-09$ & 0.605505625 \\
LotuS1-UPARSE & $1.63 \mathrm{E}-09$ & 0.611973422 \\
& & \\
\hline & & \\
\hline & BCd to the mock community \\
mothur & BCd \\
QIIME 2-Deblur & 0.430087 & \\
QIIME 2-DADA2 & 0.340823 & \\
DADA2 & 0.373356 & \\
LotuS2-DADA2 & 0.327616 & \\
LotuS2-VSEARCH & 0.35983 & \\
LotuS2-UNOISE3 & 0.324378 & \\
LotuS2-UPARSE & 0.34578 & \\
LotuS1-UPARSE & 0.324378 & \\
\hline
\end{tabular}

Supp. Table 3: Correlation and beta distance between the mock community and recommunity and the observed abundances by each pipeline. C) Bray-Curtis dissimilarity between 


\begin{tabular}{|l|c|c|c|} 
& Number of OTUs/ASVs & Number of reads & Fraction of reads assigned to TP taxa \\
\hline mothur & 370 & 144147 & 0.817443304 \\
\hline QIIME 2-Deblur & 198 & 480049 & 0.872517181 \\
\hline QIIME 2-DADA2 & 122 & 454082 & 0.882792095 \\
\hline DADA2 & 94 & 536901 & 0.922646819 \\
\hline LotuS2-DADA2 & 61 & 497970 & 0.867775167 \\
\hline LotuS2-VSEARCH & 53 & 494122 & 0.979268278 \\
\hline LotuS2-UNOISE3 & 60 & 423292 & 0.975794487 \\
\hline LotuS2-UPARSE & 53 & 494122 & 0.979268278 \\
\hline LotuS1-UPARSE & 66 & 148959 & 0.979202331 \\
\hline
\end{tabular}
genus level

911 Influence of dereplication thresholds, non-default parameters and read truncation

912 Dereplication is the pre-clustering of sequencing reads at $100 \%$ nucleotide identity, a commonly

913 used strategy to reduce the computational complexity of sequence clustering [17]. Further,

914 dereplication can be used to filter out sparsely occurring reads that could represent technical

915 artifacts, unlikely to represent true biodiversity. Therefore, LotuS2 uses a "dereplication" filter,

916 that can be user defined.

917 Overall, this filter does not mostly change the number of OTU/ASV counts, with more

918 OTUs/ASVs being recovered when the filter is more relaxed (Supp. Figure $\mathbf{2 A}, \mathbf{D}, \mathbf{G})$. This is

919 expected because this filter is designed to remove sparse OTUs/ASVs that could both represent

920 technical replicates as well as extremely rare microbes. However, this did not affect the overall

921 community reproducibility of either gut- or soil-16S samples. However, in soil-ITS samples, we

922 noted a dramatic decrease in BCd between technical replicates at stricter dereplication cut-offs

923 (Supp. Figure 2H-I).

924 The number of retrieved reads remained very stable independent of filtering stringency; this is

925 expected because the backmapping of mid-quality reads will re-introduce reads not passing the dereplication filter. 
927 LotuS2 uses several default options (-lulu 1, -xtalk 0, -keepUnclassified 0 and -ITSX 1; where

928 "1" means the option is "activated" and "0" means "deactivated"). When activated, -lulu option

929 uses LULU R package [23] to merge OTUs/ASVs based on their co-occurrences; -xtalk option

930 checks for cross-talk [32], -keepUnclassified includes unclassified (i.e. not matching to any

931 taxon in the taxonomy database) OTUs/ASVs in the final matrix and -ITSx activates the ITSx

932 program [31] to only retain OTUs fitting to ITS1/ITS2 hmm models. The impact of these

933 parameters on the reproducibility of LotuS2 was tested (Supp. Figure 3). Overall, non-default

934 options did not change the BCd between the technical replicates except -keepUnclassified 1

935 notably increasing BCd in gut-16S, while -lulu 0 slightly increased BCd in soil-ITS.

937 Read length truncation is frequently used to remove the typically low quality 3' end of reads

$938[8,17]$. This is impacting the retrieved read counts as well as observed OTU/ASV diversity. For

939 example, at 170 bp read truncation, mothur, DADA2 and QIIME 2-DADA2 were severely

940 impacted in merging read pairs, failing or only integrating a fraction of read pairs in gut and soil-

941 16S datasets Supp. Figure 4). While LotuS2 also had slightly different read and cluster

942 numbers with changing truncation lengths, it was more stable, because reads are merged in the

943 seed extension step after sequence clustering on truncated, high-quality reads are completed

944 (Supp. Figure 4). In shorter or longer read truncations, LotuS2 was still performing the best with

945 the lowest BCd (Supp. Figure 5A,C) and Jd (Supp. Figure 5B,D) between technical replicates

946 in both gut- and soil-16S datasets.

947 Taken together, the higher performance of LotuS2 in reproducibility of the dataset was

948 independent of the dereplication parameters and read truncation length. 